$\underline{\text { Preprint typeset in JHEP style - PAPER VERSION }}$

OIQP-04-2

hep-lat/0410035

\title{
Various Super Yang-Mills Theories with Exact Supersymmetry on the Lattice
}

\author{
Fumihiko Sugino \\ Okayama Institute for Quantum Physics \\ Kyoyama 1-9-1, Okayama 700-0015, Japan \\ E-mail: fumihiko_sugino@pref.okayama.jp
}

\begin{abstract}
We continue to construct lattice super Yang-Mills theories along the line discussed in the previous papers $[1,2]$. In our construction of $\mathcal{N}=2,4$ theories in four dimensions, the problem of degenerate vacua seen in [1] is resolved by extending some fields and soaking up would-be zero-modes in the continuum limit, while in the weak coupling expansion some surplus modes appear both in bosonic and fermionic sectors reflecting the exact supersymmetry. A slight modification to the models is made such that all the surplus modes are eliminated in two- and three-dimensional models obtained by dimensional reduction thereof. $\mathcal{N}=4,8$ models in three dimensions need fine-tuning of three and one parameters respectively to obtain the desired continuum theories, while two-dimensional models with $\mathcal{N}=4,8$ do not require any fine-tuning.
\end{abstract}

Keywords: Lattice Quantum Field Theory, Lattice Gauge Field Theories, Extended Supersymmetry, Topological Field Theories. 


\section{Contents}

1. Introduction 1

2. Naive 4D $\mathcal{N}=2$ Lattice Model and Its Slight Modification 3

2.1 Naive Lattice Model for $4 \mathrm{D} \mathcal{N}=2 \quad 3$

2.2 Slightly Modified Model for $4 \mathrm{D} \mathcal{N}=2 \quad 10$

3. Dimensionally Reduced $\mathcal{N}=4$ Models in Two and Three Dimensions 11

$3.13 \mathrm{D} \mathcal{N}=4$ Case 11

$3.22 \mathrm{D} \mathcal{N}=4$ Case $\quad 16$

4. Naive $4 \mathrm{D} \mathcal{N}=4$ Lattice Model and Its Slight Modification 18

$\begin{array}{ll}4.1 & \text { Naive Lattice Model for 4D } \mathcal{N}=4\end{array}$

4.2 Slightly Modified Model for $4 \mathrm{D} \mathcal{N}=4 \quad 20$

5. Dimensionally Reduced $\mathcal{N}=8$ Models in Two and Three Dimensions 21

$5.13 \mathrm{D} \mathcal{N}=8$ Case 21

$5.22 \mathrm{D} \mathcal{N}=8$ Case 24

6. Summary and Discussions 24

A. On Solutions of Eq. (2.10) 25

B. Resolution of Vacuum Degeneracy 27

C. Explicit Form of the matrices $\gamma_{\mu}, P_{\mu}, P_{\mu}^{\prime} \quad 29$

C.1 Naive Model for four-dimensional $\mathcal{N}=2$ Theory 29

C.2 Slightly Modified Model for four-dimensional $\mathcal{N}=2$ Theory 30

C.3 Naive Model for four-dimensional $\mathcal{N}=4$ Theory 30

C.4 Slightly Modified Model for four-dimensional $\mathcal{N}=4$ Theory 30

\section{Introduction}

In this paper, we continue to discuss on a lattice formulation of supersymmetric YangMills (SYM) theories with extended supersymmetries along the line of the papers $[1,2]$. The formulation is based on 'topological field theory (TFT) formulation' [3] and 'balanced topological field theory (BTFT) formulation' [4] of $\mathcal{N}=2,4$ SYM theories, respectively ${ }^{1}$. It exactly realizes one or two supercharges on the lattice which can be regarded as a

\footnotetext{
${ }^{1}$ See refs. [5] for related constructions of lattice models for theories without gauge symmetry.
} 
fermionic internal symmetry. See refs. [6]-[10] for some recent works on other approaches to lattice supersymmetry. (Also, for recent reviews, see [11].)

In ref. [1], we discussed on a general aspect of the formulation, and encountered a problem of degenerate vacua of the same kind as in [12]. There we resolved it introducing supersymmetry breaking terms which become irrelevant in the continuum limit. In [2], focusing on two-dimensional lattice SYM theories with $\mathcal{N}=2,4$, we have presented modifications to resolve the degenerated vacua without affecting the exact supersymmetry. It has been shown that in the continuum limit the two-dimensional models flow to the desired continuum theories possessing full supersymmetry and rotational invariance without fine-tuning.

Here, we first consider lattice models for four-dimensional SYM theories with $\mathcal{N}=$ 2, 4 and their dimensionally reduced versions. The problem of the vacuum degeneracy is resolved in four dimensions by extending some fields and soaking up would-be zero-modes in the continuum limit, while surplus zero-modes of the kinetic terms with nonzero momenta appear after the weak field expansion of the gauge link variables. As a manifestation of the exact supersymmetry, such modes exist in both of bosonic and fermionic sectors with the equal multiplicity. Because each of the surplus modes has smaller degrees of freedom than the ordinary zero-mode with zero-momentum, strictly speaking they are not the socalled doublers which have the degrees of freedom equivalent to those of the zero-mode with zero-momentum. Their appearence is considered to reflect the fact that our models do not exactly realize a lattice counter part of the topological term $\int \operatorname{tr} F \wedge F$. It is not a topological quantity on the lattice in the sense that an arbitrary small variation of the quantity does not always vanish, and it has some surplus dynamical modes, which we are observing as the surplus modes. In this paper, we will not pursue more on the fourdimensional models. Instead, we make use of them as an intermediate step to construct two- and three-dimensional models without surplus modes. This is a main issue of this paper. The lower-dimensional models have $\mathcal{N}=4,8$ supersymmetries in two and three dimensions, and they are obtained by dimensional reduction from the four-dimensional models after a slight modification done. The renormalization arguments based on the dimensional analysis and symmetries of the lattice action show that three-dimensional $\mathcal{N}=4,8$ cases lead the target continuum theories with tuning three and one parameters respectively, while the two-dimensional cases need no fine-tuning.

This paper is organized as follows. In section 2, we discuss a naive model for fourdimensional $\mathcal{N}=2$ SYM theory as well as its slightly modified version, and observe surplus zero-modes appearing in the kinetic terms. In section 3 , by considering dimensional reduction of the slightly modified four-dimensional model, we construct lattice models free from the surplus modes defined in two and three dimensions. Also, the renormalization arguments are presented near the continuum limit. Section 4 is devoted to both of a naive lattice model and its slightly modified one for $\mathcal{N}=4 \mathrm{SYM}$ theory in four dimensions. In section $5, \mathcal{N}=8 \mathrm{SYM}$ models on the two and three dimensional lattices are presented and their renormalization properties are examined. We summarize the result obtained so far and discuss some future directions in section 6. Discussions on the resolution of the vacuum degeneracy are presented in appendices $\mathrm{A}$ and $\mathrm{B}$. Appendix $\mathrm{C}$ gives the explicit 
form of the matrices $\gamma_{\mu}, P_{\mu}, P_{\mu}^{\prime}$ appearing in the fermion kinetic terms of various models.

Throughout this paper, we consider the gauge group $G=\mathrm{SU}(N)$ and the $d$-dimensional hypercubic lattice $\mathbf{Z}^{d}(d=2,3,4)$. Gauge fields are expressed as compact unitary variables

$$
U_{\mu}(x)=e^{i a A_{\mu}(x)}
$$

on the link $(x, x+\hat{\mu})$. ' $a$ ' stands for the lattice spacing, and $x \in \mathbf{Z}^{d}$ the lattice site. Other fields, put on the sites, are traceless hermitian matrices expanded by the $\mathrm{SU}(N)$ generators (the complex scalars $\phi, \bar{\phi}$ are complexified versions of them ). We extend some fields to hermitian matrices with nonvanishing trace parts, which are expressed by putting the hat $\left({ }^{\wedge}\right)$.

\section{Naive $4 \mathrm{D} \mathcal{N}=2$ Lattice Model and Its Slight Modification}

We consider a naive lattice model for four-dimensional $\mathcal{N}=2$ SYM theory as well as its slightly modified version, which are almost same as the model presented in ref. [1]. Notations are same as in [1].

\subsection{Naive Lattice Model for $4 \mathrm{D} \mathcal{N}=2$}

We start with the lattice action for four-dimensional $\mathcal{N}=2$ :

$$
\begin{aligned}
S_{4 D \mathcal{N}=2}^{\mathrm{LAT}}=Q \frac{1}{2 g_{0}^{2}} \sum_{x} \operatorname{tr} & {\left[\frac{1}{4} \eta(x)[\phi(x), \bar{\phi}(x)]-i \vec{\chi}(x) \cdot(\vec{\Phi}(x)+\Delta \vec{\Phi}(x))+\vec{\chi}(x) \cdot \vec{H}(x)\right.} \\
& \left.+i \sum_{\mu=1}^{4} \psi_{\mu}(x)\left(\bar{\phi}(x)-U_{\mu}(x) \bar{\phi}(x+\hat{\mu}) U_{\mu}(x)^{\dagger}\right)\right]
\end{aligned}
$$

where $\vec{H}, \vec{\chi}, \vec{\Phi}, \Delta \vec{\Phi}$ are three-component vectors. In terms of the plaquette variables

$$
U_{\mu \nu}(x) \equiv U_{\mu}(x) U_{\nu}(x+\hat{\mu}) U_{\mu}(x+\hat{\nu})^{\dagger} U_{\nu}(x)^{\dagger}
$$

$\vec{\Phi}(x)$ and $\Delta \vec{\Phi}(x)$ are expressed as

$$
\begin{aligned}
\Phi_{1}(x) & =-i\left[U_{14}(x)-U_{41}(x)+U_{23}(x)-U_{32}(x)\right], \\
\Phi_{2}(x) & =-i\left[U_{24}(x)-U_{42}(x)+U_{31}(x)-U_{13}(x)\right], \\
\Phi_{3}(x) & =-i\left[U_{34}(x)-U_{43}(x)+U_{12}(x)-U_{21}(x)\right], \\
\Delta \Phi_{1}(x) & =-r\left[W_{14}(x)+W_{23}(x)\right], \\
\Delta \Phi_{2}(x) & =-r\left[W_{24}(x)+W_{31}(x)\right], \\
\Delta \Phi_{3}(x) & =-r\left[W_{34}(x)+W_{12}(x)\right],
\end{aligned}
$$

with

$$
W_{\mu \nu}(x) \equiv 2-U_{\mu \nu}(x)-U_{\nu \mu}(x) .
$$

$\phi, \bar{\phi}$ represent complex scalar fields, and $\psi_{\mu}, \vec{\chi}, \eta$ fermions. Also, $\vec{H}$ are auxiliary fields. The nilpotent supersymmetry $Q$ :

$$
Q^{2}=(\text { infinitesimal gauge transformation with the parameter } \phi \text { ) }
$$


acts to the lattice fields as

$$
\begin{aligned}
& Q U_{\mu}(x)=i \psi_{\mu}(x) U_{\mu}(x), \\
& Q \psi_{\mu}(x)=i \psi_{\mu}(x) \psi_{\mu}(x)-i\left(\phi(x)-U_{\mu}(x) \phi(x+\hat{\mu}) U_{\mu}(x)^{\dagger}\right), \\
& Q \phi(x)=0, \\
& Q \vec{\chi}(x)=\vec{H}(x), \quad Q \vec{H}(x)=[\phi(x), \vec{\chi}(x)], \\
& Q \bar{\phi}(x)=\eta(x), \quad Q \eta(x)=[\phi(x), \bar{\phi}(x)] .
\end{aligned}
$$

The action has the $Q$ exact form based on the 'TFT formulation' [3] of four-dimensional $\mathcal{N}=2$ SYM discussed in ref. [1]. This $Q$, preserved at the lattice level, is one of eight supercharges of four-dimensional $\mathcal{N}=2$ supersymmetry. The action has the global $\mathrm{U}(1)_{R}$ internal symmetry:

$$
\begin{aligned}
& \psi_{\mu}(x) \rightarrow e^{i \Lambda} \psi_{\mu}(x), \quad \vec{\chi}(x) \rightarrow e^{-i \Lambda} \vec{\chi}(x), \quad \eta(x) \rightarrow e^{-i \Lambda} \eta(x), \\
& \phi(x) \rightarrow e^{i 2 \Lambda} \phi(x), \quad \bar{\phi}(x) \rightarrow e^{-i 2 \Lambda} \bar{\phi}(x)
\end{aligned}
$$

with other fields intact.

Note that we employ dimensionless variables on the lattice, and thus various quantities are of the following orders:

$$
\begin{aligned}
& \psi_{\mu}(x), \vec{\chi}(x), \eta(x)=O\left(a^{3 / 2}\right), \quad \phi(x), \bar{\phi}(x)=O(a), \quad \vec{H}(x)=O\left(a^{2}\right), \\
& Q=O\left(a^{1 / 2}\right) .
\end{aligned}
$$

The action (2.1) takes the almost same form as the one presented in the paper [1] except the terms containing $\Delta \vec{\Phi}(x)$. The result of the $Q$-action in (2.1) becomes

$$
\begin{aligned}
S_{4 D \mathcal{N}=2}^{\mathrm{LAT}}= & \frac{1}{2 g_{0}^{2}} \sum_{x} \operatorname{tr}\left[\frac{1}{4}[\phi(x), \bar{\phi}(x)]^{2}+\vec{H}(x) \cdot \vec{H}(x)-i \vec{H}(x) \cdot(\vec{\Phi}(x)+\Delta \vec{\Phi}(x))\right. \\
& +\sum_{\mu=1}^{4}\left(\phi(x)-U_{\mu}(x) \phi(x+\hat{\mu}) U_{\mu}(x)^{\dagger}\right)\left(\bar{\phi}(x)-U_{\mu}(x) \bar{\phi}(x+\hat{\mu}) U_{\mu}(x)^{\dagger}\right) \\
& -\frac{1}{4} \eta(x)[\phi(x), \eta(x)]-\vec{\chi}(x) \cdot[\phi(x), \vec{\chi}(x)] \\
& -\sum_{\mu=1}^{4} \psi_{\mu}(x) \psi_{\mu}(x)\left(\bar{\phi}(x)+U_{\mu}(x) \bar{\phi}(x+\hat{\mu}) U_{\mu}(x)^{\dagger}\right)+i \vec{\chi}(x) \cdot Q(\vec{\Phi}(x)+\Delta \vec{\Phi}(x)) \\
& \left.-i \sum_{\mu=1}^{4} \psi_{\mu}(x)\left(\eta(x)-U_{\mu}(x) \eta(x+\hat{\mu}) U_{\mu}(x)^{\dagger}\right)\right]
\end{aligned}
$$

Note that since $\vec{H}$ is traceless, the classical vacua are determined by

$$
\vec{\Phi}(x)+\Delta \vec{\Phi}(x)-\left(\frac{1}{N} \operatorname{tr}[\vec{\Phi}(x)+\Delta \vec{\Phi}(x)]\right) \mathbf{1}_{N}=0 .
$$

As seen in appendix A, however its solutions are not unique and distribute continuously from $U_{\mu \nu}(x)=1$. Thus, a manipulation using the admissibility conditions would not work to single out the minimum $U_{\mu \nu}(x)=1$ as in [2]. 
On the other hand, the equation

$$
\Phi(x)+\Delta \Phi(x)=0
$$

for $G=\mathrm{SU}(N)$ has the unique solution $U_{\mu \nu}(x)=1$ with appropriately chosen $r$ in the range ${ }^{2}$

$$
r=\cot \varphi: \quad 0<\varphi \leq \frac{\pi}{2 N}
$$

as shown in appendix B.

Here, we extend $\vec{\chi}(x), \vec{H}(x)$ to the hermitian matrices $\overrightarrow{\hat{\chi}}(x), \overrightarrow{\hat{H}}(x)$ with nonzero trace parts to introduce the variables $\vec{\chi}^{(0)}(x), \vec{H}^{(0)}(x)$ proportional to the unit matrix:

$$
\begin{array}{lr}
\vec{\chi}^{(0)}(x)=\underline{\vec{\chi}}^{(0)}(x) \mathbf{1}_{N}, \quad \vec{H}^{(0)}(x)=\underline{\vec{H}}^{(0)}(x) \mathbf{1}_{N} \\
\overrightarrow{\hat{H}}(x)=\vec{H}(x)+\vec{H}^{(0)}(x), & \overrightarrow{\hat{\chi}}(x)=\vec{\chi}(x)+\vec{\chi}^{(0)}(x) .
\end{array}
$$

The fields with the uniderline mean the coefficients proportional to the unit matrix. The $Q$-transformation (2.6) of $\vec{\chi}(x)$ and $\vec{H}(x)$ is naturally extended to

$$
Q \overrightarrow{\hat{\chi}}(x)=\overrightarrow{\hat{H}}(x), \quad Q \overrightarrow{\hat{H}}(x)=[\phi(x), \overrightarrow{\hat{\chi}}(x)]
$$

with

$$
Q \vec{\chi}^{(0)}(x)=\vec{H}^{(0)}(x), \quad Q \vec{H}^{(0)}(x)=0 .
$$

The lattice action is modified as

$$
\begin{aligned}
\hat{S}_{4 D \mathcal{N}=2}^{\mathrm{LAT}}=Q \frac{1}{2 g_{0}^{2}} \sum_{x} \operatorname{tr}\left[\frac{1}{4} \eta(x)[\phi(x), \bar{\phi}(x)]-i \overrightarrow{\hat{\chi}}(x) \cdot(\vec{\Phi}(x)+\Delta \vec{\Phi}(x))+\overrightarrow{\hat{\chi}}(x) \cdot \overrightarrow{\hat{H}}(x)\right. & \\
& \left.\quad+i \sum_{\mu=1}^{2} \psi_{\mu}(x)\left(\bar{\phi}(x)-U_{\mu}(x) \bar{\phi}(x+\hat{\mu}) U_{\mu}(x)^{\dagger}\right)\right] \\
=\frac{1}{2 g_{0}^{2}} \sum_{x} \operatorname{tr} & {\left[\frac{1}{4}[\phi(x), \bar{\phi}(x)]^{2}+\overrightarrow{\hat{H}}(x)^{2}-i \overrightarrow{\hat{H}}(x) \cdot(\vec{\Phi}(x)+\Delta \vec{\Phi}(x))\right.} \\
+ & \sum_{\mu=1}^{2}\left(\phi(x)-U_{\mu}(x) \phi(x+\hat{\mu}) U_{\mu}(x)^{\dagger}\right)\left(\bar{\phi}(x)-U_{\mu}(x) \bar{\phi}(x+\hat{\mu}) U_{\mu}(x)^{\dagger}\right) \\
& -\frac{1}{4} \eta(x)[\phi(x), \eta(x)]-\vec{\chi}(x) \cdot[\phi(x), \vec{\chi}(x)] \\
& -\sum_{\mu=1}^{2} \psi_{\mu}(x) \psi_{\mu}(x)\left(\bar{\phi}(x)+U_{\mu}(x) \bar{\phi}(x+\hat{\mu}) U_{\mu}(x)^{\dagger}\right) \\
+ & i \overrightarrow{\hat{\chi}}(x) \cdot Q(\vec{\Phi}(x)+\Delta \vec{\Phi}(x)) \\
& \left.-i \sum_{\mu=1}^{2} \psi_{\mu}(x)\left(\eta(x)-U_{\mu}(x) \eta(x+\hat{\mu}) U_{\mu}(x)^{\dagger}\right)\right] .
\end{aligned}
$$

\footnotetext{
${ }^{2}$ In appendix B, we prove the unique solution $U_{\mu \nu}(x)=1$ for the equations $\vec{\Phi}(x)+\Delta \vec{\Phi}(x)=0$ in the case limited to (2.12). A priori, the argument itself does not exclude the possibility of the unique solution for the parameter $r$ outside of the range (2.12).
} 
Due to the trace part of $\overrightarrow{\hat{H}}(x)$, the minimum of the gauge part is uniquely determined by (2.11) with $r=\cot \varphi$ satisfying (2.12). On the other hand, the kinetic term of $\overrightarrow{\hat{\chi}}(x)$ becomes

$$
\frac{1}{2 g_{0}^{2}} \sum_{x}\left\{\operatorname{tr}[i \vec{\chi}(x) \cdot Q(\vec{\Phi}(x)+\Delta \vec{\Phi}(x))]+i \underline{\vec{z}}^{(0)}(x) \cdot Q \operatorname{tr}(\vec{\Phi}(x)+\Delta \vec{\Phi}(x))\right\} .
$$

Since the second term in the brace is of the order $O\left(a^{6}\right)$, it vanishes in the contiuum limit and $\underline{\vec{\chi}}^{(0)}(x)$ become fermion zero-modes. If we integrate out $\underline{\vec{x}}^{(0)}(x)$, we will have the nontrivial constraints

$$
0=Q \operatorname{tr}(\vec{\Phi}(x)+\Delta \vec{\Phi}(x))
$$

leading

$$
\begin{aligned}
& 0=\operatorname{tr}\left[F_{14}(x)\left(D_{1} \psi_{4}(x)-D_{4} \psi_{1}(x)\right)+F_{23}(x)\left(D_{2} \psi_{3}(x)-D_{3} \psi_{2}(x)\right)\right] \\
& 0=\operatorname{tr}\left[F_{24}(x)\left(D_{2} \psi_{4}(x)-D_{4} \psi_{2}(x)\right)+F_{31}(x)\left(D_{3} \psi_{1}(x)-D_{1} \psi_{3}(x)\right)\right] \\
& 0=\operatorname{tr}\left[F_{34}(x)\left(D_{3} \psi_{4}(x)-D_{4} \psi_{3}(x)\right)+F_{12}(x)\left(D_{1} \psi_{2}(x)-D_{2} \psi_{1}(x)\right)\right]
\end{aligned}
$$

at the nontrivial leading order $O\left(a^{9 / 2}\right)$ in the continuum. Because such constraints are not imposed in the target continuum theory, we should avoid obtaining them. In order to do so, we soak up the would-be fermion zero-modes in the path-integral to consider the measure

$$
\mathrm{d} \mu_{\mathcal{N}=2} \equiv \mathrm{d} \mu_{\mathrm{SU}(N) \mathcal{N}=2} \prod_{x, \mathrm{~A}}\left(\mathrm{~d} \underline{H}_{\mathrm{A}}^{(0)}(x) \mathrm{d} \underline{\chi}_{\mathrm{A}}^{(0)}(x) \underline{\chi}_{\mathrm{A}}^{(0)}(x)\right)
$$

with $\mathrm{d} \mu_{\mathrm{SU}(N) \mathcal{N}=2}$ being the measure for the $\mathrm{SU}(N)$ variables. Note that $\mathrm{d} \underline{\chi}_{\mathrm{A}}^{(0)}(x) \underline{\chi}_{\mathrm{A}}^{(0)}(x)$ is $\mathrm{U}(1)_{R}$ invariant, because $\mathrm{d} \underline{\chi}_{\mathrm{A}}^{(0)}(x)$ transforms same as the derivative $\partial / \partial \underline{\chi}_{\mathrm{A}}^{(0)}(x)$. However, the insertion of the would-be zero-modes violates the $Q$ invariance as

$$
Q\left(\mathrm{~d} \underline{H}_{\mathrm{A}}^{(0)}(x) \mathrm{d} \underline{\chi}_{\mathrm{A}}^{(0)}(x) \underline{\chi}_{\mathrm{A}}^{(0)}(x)\right)=-\mathrm{d} \underline{H}_{\mathrm{A}}^{(0)}(x) \mathrm{d} \underline{\chi}_{\mathrm{A}}^{(0)}(x) \underline{H}_{\mathrm{A}}^{(0)}(x),
$$

although the action (2.16) is manifestly $Q$ invariant.

Here we consider the observables consisting the operators in the $\mathrm{SU}(N)$ sector i.e. independent of $\vec{H}^{(0)}(x)$ and $\vec{\chi}^{(0)}(x)$. Let us write the action as

$$
\begin{aligned}
& \hat{S}_{4 D \mathcal{N}=2}^{\mathrm{LAT}}=S_{4 D \mathcal{N}=2}^{\mathrm{LAT}}+\frac{N}{2 g_{0}^{2}} \sum_{x} {\left[\underline{\vec{H}}^{(0)}(x)^{2}-i \underline{\vec{H}}^{(0)}(x) \cdot \frac{1}{N} \operatorname{tr}(\vec{\Phi}(x)+\Delta \vec{\Phi}(x))\right.} \\
&\left.+i \underline{\vec{\chi}}^{(0)}(x) \cdot Q \frac{1}{N} \operatorname{tr}(\vec{\Phi}(x)+\Delta \vec{\Phi}(x))\right],
\end{aligned}
$$

so that the dependence of $\underline{\vec{H}}^{(0)}(x)$ and $\underline{\vec{\chi}}^{(0)}(x)$ can be explicitly seen. From $(2.22),(2.23)$, the $Q$-transformation of $\mathrm{d} \mu_{\mathcal{N}=2} e^{-\hat{S}_{4 D \mathcal{N}=2}^{\mathrm{LAT}}}$ leads

$$
\begin{aligned}
& \int Q\left(\mathrm{~d} \mu_{\mathcal{N}=2} e^{-\hat{S}_{4 D \mathcal{N}=2}^{\mathrm{LAT}}}\right)=\int \mathrm{d} \mu_{\mathrm{SU}(N) \mathcal{N}=2} e^{-S_{\mathcal{N}=2}^{\mathrm{LAT}}}\left(\prod_{x, \mathrm{~A}} \underline{\mathrm{d}}_{\mathrm{A}}^{(0)}(x)\right) \\
& \times \sum_{x}\left[\frac{i}{2 g_{0}^{2}} \underline{\vec{H}}^{(0)}(x) \cdot Q \operatorname{tr}(\vec{\Phi}(x)+\Delta \vec{\Phi}(x))\right] \\
& \times \exp \left\{-\frac{N}{2 g_{0}^{2}} \sum_{x}\left[\underline{\vec{H}}^{(0)}(x)^{2}-i \underline{\vec{H}}^{(0)}(x) \cdot \frac{1}{N} \operatorname{tr}(\vec{\Phi}(x)+\Delta \vec{\Phi}(x))\right]\right\}
\end{aligned}
$$


after integrating out $\underline{\vec{\chi}}^{(0)}$. As the result of the integration of $\underline{\vec{H}}^{(0)}$, we obtain

$$
\begin{aligned}
\int Q\left(\mathrm{~d} \mu_{\mathcal{N}=2} e^{\left.-\hat{S}_{4 D \mathcal{N}=2}^{\mathrm{LAT}}\right)=}\right. & \int \mathrm{d} \mu_{\mathrm{SU}(N) \mathcal{N}=2} e^{-S_{\mathcal{N}=2}^{\mathrm{LAT}}} e^{-\frac{1}{8 N g_{0}^{2}} \sum_{x}[\operatorname{tr}(\vec{\Phi}(x)+\Delta \vec{\Phi}(x))]^{2}} \\
& \times Q \sum_{x} \frac{-1}{8 N g_{0}^{2}}[\operatorname{tr}(\vec{\Phi}(x)+\Delta \vec{\Phi}(x))]^{2}
\end{aligned}
$$

which means that the insertion of the would-be fermion zero-modes is equivalent to adding the supersymmetry breaking term

$$
\Delta S=\frac{1}{8 N g_{0}^{2}} \sum_{x}[\operatorname{tr}(\vec{\Phi}(x)+\Delta \vec{\Phi}(x))]^{2}
$$

to the $Q$ invariant action $S_{4 D \mathcal{N}=2}^{\mathrm{LAT}}(2.1) . \Delta S$ supplies the trace part of $\vec{\Phi}(x)+\Delta \vec{\Phi}(x)$ leading the condition for the minima (2.11) to resolve the degeneracy. In the continuum limit, $\Delta S$ becomes irrelevant, because $\operatorname{tr}(\vec{\Phi}(x)+\Delta \vec{\Phi}(x))$ is of the order $O\left(a^{4}\right)$.

Surplus Modes The action (2.16), equivalently to $S_{4 D \mathcal{N}=2}^{\mathrm{LAT}}+\Delta S$, resolves the vacuum degeneracy to fix the single vacuum $U_{\mu \nu}(x)=1$. Because $U_{\mu \nu}(x)=1$ is equivalent to $U_{\mu}(x)=1$ modulo gauge transformations on the infinite lattice $x \in \mathbf{Z}^{4}$, the expansion

$$
U_{\mu}(x)=1+i a A_{\mu}(x)+\cdots
$$

is justified to treat fluctuations around the minimum.

After the weak field expansion (2.27), the fermion kinetic terms are expressed as

$$
\begin{aligned}
S_{f}^{(2)} & =\frac{a^{4}}{2 g_{0}^{2}} \sum_{x, \mu} \operatorname{tr}\left[-\frac{1}{2} \Psi(x)^{T}\left(P_{\mu}+\gamma_{\mu}\right) \Delta_{\mu} \Psi(x)+\frac{1}{2} \Psi(x)^{T}\left(P_{\mu}-\gamma_{\mu}\right) \Delta_{\mu}^{*} \Psi(x)\right] \\
& =\frac{a^{4}}{2 g_{0}^{2}} \sum_{x, \mu} \operatorname{tr}\left[-\frac{1}{2} \Psi(x)^{T} \gamma_{\mu}\left(\Delta_{\mu}+\Delta_{\mu}^{*}\right) \Psi(x)-a \frac{1}{2} \Psi(x)^{T} P_{\mu} \Delta_{\mu} \Delta_{\mu}^{*} \Psi(x)\right]
\end{aligned}
$$

where the fermion fields were rescaled by $a^{3 / 2}$ as indicated in (2.8) and combined as

$$
\Psi^{T}=\left(\psi_{1}, \cdots, \psi_{4}, \chi_{1}, \chi_{2}, \chi_{3}, \frac{1}{2} \eta\right)
$$

The transpose operation acts only on the spinor indices. $\Delta_{\mu}$ and $\Delta_{\mu}^{*}$ represent forward and backward difference operators respectively:

$$
\Delta_{\mu} f(x) \equiv \frac{1}{a}(f(x+\hat{\mu})-f(x)), \quad \Delta_{\mu}^{*} f(x) \equiv \frac{1}{a}(f(x)-f(x-\hat{\mu})) .
$$

The explicit form of the matrices $\gamma_{\mu}, P_{\mu}$ is given in appendix C.1. They satisfy

$$
\left\{\gamma_{\mu}, \gamma_{\nu}\right\}=-2 \delta_{\mu \nu}, \quad\left\{P_{\mu}, P_{\nu}\right\}=2 \delta_{\mu \nu}, \quad\left\{\gamma_{\mu}, P_{\mu}\right\}=0
$$

while $\gamma_{\mu}$ and $P_{\nu}$ with $\mu \neq \nu$ do not anticommute:

$$
\left\{\gamma_{\mu}, P_{\nu}\right\} \neq 0 \text { for } \mu \neq \nu
$$


Notice its difference from the two-dimensional $\mathcal{N}=2,4$ cases in the paper [2], where

$$
\left\{\gamma_{\mu}, P_{\nu}\right\}=0 \quad \text { for } \quad \forall \mu, \nu=1,2
$$

leads no fermion doublers.

The kernel of the kinetic term (2.28) is written in the momentum space $-\pi / a \leq q_{\mu}<$ $\pi / a$ as

$$
\tilde{D}(q)=\sum_{\mu=1}^{4}\left[-i \gamma_{\mu} \frac{1}{a} \sin \left(q_{\mu} a\right)+2 P_{\mu} \frac{1}{a} \sin ^{2}\left(\frac{q_{\mu} a}{2}\right)\right] .
$$

Due to the property $(2.32)$, it is not clear if $\tilde{D}(q)$ has zero-modes only at the origin $\left(q_{1}, \cdots, q_{4}\right)=(0, \cdots, 0)$. Indeed, $\tilde{D}(q)$ has zero-eigenvalues at the points

$$
\begin{aligned}
& \left(q_{1}, q_{2}, q_{3}, q_{4}\right)=(0,0,0,0), \quad\left( \pm \frac{\pi}{2 a}, \mp \frac{\pi}{2 a}, 0,0\right), \quad\left( \pm \frac{\pi}{2 a}, 0, \mp \frac{\pi}{2 a}, 0\right), \quad\left( \pm \frac{\pi}{2 a}, 0,0, \mp \frac{\pi}{2 a}\right), \\
& \left(0, \pm \frac{\pi}{2 a}, \mp \frac{\pi}{2 a}, 0\right), \quad\left(0, \pm \frac{\pi}{2 a}, 0, \mp \frac{\pi}{2 a}\right), \quad\left(0,0, \pm \frac{\pi}{2 a}, \mp \frac{\pi}{2 a}\right), \\
& \left( \pm \frac{\pi}{2 a}, \mp \frac{\pi}{2 a}, \pm \frac{\pi}{2 a}, \mp \frac{\pi}{2 a}\right), \quad\left( \pm \frac{\pi}{2 a}, \pm \frac{\pi}{2 a}, \mp \frac{\pi}{2 a}, \mp \frac{\pi}{2 a}\right), \quad\left( \pm \frac{\pi}{2 a}, \mp \frac{\pi}{2 a}, \mp \frac{\pi}{2 a}, \pm \frac{\pi}{2 a}\right), \\
& \left( \pm \frac{\pi}{3 a}, \pm \frac{\pi}{3 a}, \pm \frac{\pi}{3 a}, \mp \frac{2 \pi}{3 a}\right), \quad\left( \pm \frac{\pi}{3 a}, \pm \frac{\pi}{3 a}, \mp \frac{2 \pi}{3 a}, \pm \frac{\pi}{3 a}\right), \quad\left( \pm \frac{\pi}{3 a}, \mp \frac{2 \pi}{3 a}, \pm \frac{\pi}{3 a}, \pm \frac{\pi}{3 a}\right), \\
& \left(\mp \frac{2 \pi}{3 a}, \pm \frac{\pi}{3 a}, \pm \frac{\pi}{3 a}, \pm \frac{\pi}{3 a}\right) .
\end{aligned}
$$

The modes other than $\left(q_{1}, \cdots, q_{4}\right)=(0, \cdots, 0)$ are surplus, and we do not want them in order to get the desired continuum theory. Since the surplus modes are not exact zero-modes of the full action (they are only of the quadratic term), for each of them a corresponding supersymmetric partner exists in the bosonic sector in consequence of the exact supersymmetry $Q$. (In the weak field expansion (2.27), the effect of $\Delta S$ is irrelevant.)

Let us see the bosonic surplus modes. We may consider quadratic terms of $A_{\mu}(x)$ in $\operatorname{tr}[\vec{\Phi}(x)+\Delta \vec{\Phi}(x)]^{2}$ after the expansion (2.27), i.e. linear terms of $A_{\mu}(x)$ in $\vec{\Phi}(x)$. Note that terms from $\Delta \vec{\Phi}(x)$ yield irrelevant interaction terms instead of the gauge kinetic term, and we can neglect them in the analysis here. In terms of the Fourier transformed basis

$$
\Phi_{\mathrm{A}}(x)=\int_{-\pi / a}^{\pi / a} \frac{\mathrm{d}^{4} q}{(2 \pi)^{4}} e^{i a q \cdot x} \tilde{\Phi}_{\mathrm{A}}(q),
$$

$\vec{\Phi}$ becomes

$$
\begin{aligned}
\tilde{\Phi}_{1}(q)= & 2 a\left[\left(e^{i a q_{1}}-1\right) \tilde{A}_{4}(q)-\left(e^{i a q_{4}}-1\right) \tilde{A}_{1}(q)+\left(e^{i a q_{2}}-1\right) \tilde{A}_{3}(q)-\left(e^{i a q_{3}}-1\right) \tilde{A}_{2}(q)\right] \\
& +O\left(\tilde{A}^{2}\right) \\
\tilde{\Phi}_{2}(q)= & 2 a\left[\left(e^{i a q_{2}}-1\right) \tilde{A}_{4}(q)-\left(e^{i a q_{4}}-1\right) \tilde{A}_{2}(q)+\left(e^{i a q_{3}}-1\right) \tilde{A}_{1}(q)-\left(e^{i a q_{1}}-1\right) \tilde{A}_{3}(q)\right] \\
& +O\left(\tilde{A}^{2}\right) \\
\tilde{\Phi}_{3}(q)= & 2 a\left[\left(e^{i a q_{3}}-1\right) \tilde{A}_{4}(q)-\left(e^{i a q_{4}}-1\right) \tilde{A}_{3}(q)+\left(e^{i a q_{1}}-1\right) \tilde{A}_{2}(q)-\left(e^{i a q_{2}}-1\right) \tilde{A}_{1}(q)\right] \\
& +O\left(\tilde{A}^{2}\right) .
\end{aligned}
$$


We shall see that there appear at the momenta $(2.35)$ the zero-modes $\tilde{A}_{\mu}(q)$ satisfying

$$
\overrightarrow{\tilde{\Phi}}(q)=0+O\left(\tilde{A}^{2}\right)
$$

The case $q=0$ is trivial. For the rest, as an example, let us consider the case $q_{(12)} \equiv$ $\left(\frac{\pi}{2 a},-\frac{\pi}{2 a}, 0,0\right)$. The solution of $(2.38)$ is

$$
\left(\tilde{A}_{1}^{\alpha}\left(q_{(12)}\right), \tilde{A}_{2}^{\alpha}\left(q_{(12)}\right), \tilde{A}_{3}^{\alpha}\left(q_{(12)}\right), \tilde{A}_{4}^{\alpha}\left(q_{(12)}\right)\right)=\left(K^{\alpha}, i K^{\alpha}, L^{\alpha},-i L^{\alpha}\right)
$$

with $\alpha=1, \cdots, N^{2}-1$ being the index of a basis of the gauge generators and $K^{\alpha}, L^{\alpha}$ complex numbers. Because $K^{\alpha}$ is absorbed by the linearized gauge transformation

$$
\delta \tilde{A}_{\mu}^{\alpha}\left(q_{(12)}\right)=\left(e^{i a q_{(12) \mu}}-1\right) \frac{1}{1-i} K^{\alpha},
$$

only one degree of freedom $L^{\alpha}$ is dynamical. Correspondingly, the Dirac operator (2.34) at $q=q_{(12)}$ has the two eigenvectors for zero-eigenvalue:

$$
(0,0,1,-i, 0,0,0,0)^{T}, \quad(0,0,0,0,1,-i, 0,0)^{T}
$$

meaning one on-shell degree of freedom for fermions. Hence, bosonic and fermionic degrees of freedom for the surplus mode $q=q_{(12)}$ are balanced $^{3}$. The analysis for the other modes is similarly given.

The appearence of the surplus modes seems related to exact realization of a lattice counterpart of the topological term $\int \mathrm{d}^{4} x \varepsilon_{\mu \nu \lambda \rho} \operatorname{tr} F_{\mu \nu}(x) F_{\lambda \rho}(x)$. Writing as

$$
\begin{aligned}
& \Phi_{1}(x)+\Delta \Phi_{1}(x)=\mathcal{F}_{14}(x)+\mathcal{F}_{23}(x), \\
& \Phi_{2}(x)+\Delta \Phi_{2}(x)=\mathcal{F}_{24}(x)+\mathcal{F}_{31}(x), \\
& \Phi_{3}(x)+\Delta \Phi_{3}(x)=\mathcal{F}_{34}(x)+\mathcal{F}_{12}(x), \\
& \mathcal{F}_{\mu \nu}(x) \equiv-i\left(U_{\mu \nu}(x)-U_{\nu \mu}(x)\right)-r W_{\mu \nu}(x),
\end{aligned}
$$

$\mathcal{F}_{\mu \nu}(x)$ reduce the continuum field strengths $F_{\mu \nu}(x)$ in the limit $a \rightarrow 0$. It is easy to see that the surplus modes do not arise from $\operatorname{tr} \mathcal{F}_{\mu \nu}(x)^{2}$ in the weak field expansion (2.27) of $\operatorname{tr}[\vec{\Phi}(x)+\Delta \vec{\Phi}(x)]^{2}$, which suggests that the cross term

$$
\operatorname{tr} \mathcal{F}_{14}(x) \mathcal{F}_{23}(x)+\operatorname{tr} \mathcal{F}_{24}(x) \mathcal{F}_{31}(x)+\operatorname{tr} \mathcal{F}_{34}(x) \mathcal{F}_{12}(x)
$$

contains extra dynamical degrees of freedom corresponding to the surplus modes. Thus it is not exactly topological at the lattice level in the sense that an arbitrary small variation

\footnotetext{
${ }^{3}$ The on-shell degrees of freedom of the mode $q=q_{(12)}$ are smaller compared with the zero-mode $q=0$ having two on-shell degrees of freedom. Thus, we consider the mode $q=q_{(12)}$ not identical with the so-called doublers, and use the term "surplus modes". If it was the doubler, the Dirac operator $\tilde{D}\left(q_{(12)}\right)$ would become the zero matrix. However, it is not the case. $\tilde{D}\left(q_{(12)}\right)$ has two zero-eigenvalues as well as six nonzero-eigenvalues. The situation is same for the other surplus modes. The notion of the surplus modes is broader than that of the doublers. Nonexistence of the surplus modes leads nonexistence of the doublers, but the reverse does not hold.
} 
of the quantity does not always vanish. For the case of the gauge group U(1), the topological term has been rigorously constructed on the lattice in refs. [13]. Extension of the construction to the nonabelian case would be quite helpful to resolve our problem.

Due to the supersymmetric property, it might seem that bosonic and fermionic contributions to the surplus modes cancel each other among loop diagrams in the perturbation theory, and thus they might be harmless as long as considering observables which do not allow the surplus modes to propagate from the corresponding external lines. In order to clarify it, however we need an explicit analysis of the lattice perturbation theory. Because it is not a main issue of this paper, we will examine the above speculation elsewhere.

Note that our model realizes the $\mathrm{U}(1)_{R}$ symmetry (2.7) instead of the fermion number conservation at the lattice level. Anomaly will be induced with respect to the fermion number conservation that is broken by the lattice artifact. The situation is opposite to the conventional treatment of the four-dimensional $\mathcal{N}=2 \mathrm{SYM}$ theory (For example, see [14].), where $\mathrm{U}(1)_{R}$ becomes anomalous and the fermion number symmetry $\mathrm{U}(1)_{J}$ is preserved at the quantum level. In order to remedy it and to reproduce the $\mathrm{U}(1)_{R}$ anomaly correctly, we will have to introduce suitable counter terms to the lattice action to cancel the fermion number anomaly. In this paper, we do not pursue the four-dimensional model itself further. Instead, it is utilized as an intermediate step to construct lower-dimensional models not containing surplus modes, after making a slight modification to the four-dimensional model in the next subsection. This is a main issue of this paper.

\subsection{Slightly Modified Model for $4 \mathrm{D} \mathcal{N}=2$}

We slightly modify the action $S_{4 D \mathcal{N}=2}^{\mathrm{LAT}}+\Delta S$ with the replacement $\vec{\Phi} \rightarrow \vec{\Phi}^{\prime}, \Delta \vec{\Phi} \rightarrow \Delta \vec{\Phi}^{\prime}$, where

$$
\begin{aligned}
\Phi_{1}^{\prime}(x) & \equiv-i\left[U_{4,-1}(x)-U_{-1,4}(x)+U_{23}(x)-U_{32}(x)\right], \\
\Phi_{2}^{\prime}(x) & \equiv-i\left[U_{4,-2}(x)-U_{-2,4}(x)+U_{31}(x)-U_{13}(x)\right], \\
\Phi_{3}^{\prime}(x) & \equiv-i\left[U_{4,-3}(x)-U_{-3,4}(x)+U_{12}(x)-U_{21}(x)\right], \\
\Delta \Phi_{1}^{\prime}(x) & \equiv-r\left[W_{4,-1}(x)+W_{23}(x)\right], \\
\Delta \Phi_{2}^{\prime}(x) & \equiv-r\left[W_{4,-2}(x)+W_{31}(x)\right], \\
\Delta \Phi_{3}^{\prime}(x) & \equiv-r\left[W_{4,-3}(x)+W_{12}(x)\right] .
\end{aligned}
$$

The parameter $r$ is chosen in the range (2.12) again. The link variables along the $-\mu$ direction are defined as

$$
U_{-\mu}(x) \equiv U_{\mu}(x-\hat{\mu})^{\dagger},
$$

and then plaquettes $U_{\mu \nu}(x)$ for $\mu, \nu= \pm 1, \cdots, \pm 4$ are expressed as

$$
U_{\mu \nu}(x)=U_{\mu}(x) U_{\nu}(x+\hat{\mu}) U_{-\mu}(x+\hat{\mu}+\hat{\nu}) U_{-\nu}(x+\hat{\nu}) \quad(\mu, \nu= \pm 1, \cdots, \pm 4) .
$$

The fermion kinetic term of the modified model takes the form

$$
S_{f}^{(2)}=\frac{a^{4}}{2 g_{0}^{2}} \sum_{x} \operatorname{tr}\left[\sum_{\mu=1}^{4}\left\{-\frac{1}{2} \Psi(x)^{T} \gamma_{\mu}\left(\Delta_{\mu}+\Delta_{\mu}^{*}\right) \Psi(x)-a \frac{1}{2} \Psi(x)^{T} P_{\mu}^{\prime} \Delta_{\mu} \Delta_{\mu}^{*} \Psi(x)\right\}\right.
$$




$$
\left.+i a \sum_{\mathrm{A}=1}^{3}\left(\chi_{\mathrm{A}}(x) \Delta_{4} \Delta_{\mathrm{A}}^{*} \psi_{\mathrm{A}}(x)-\psi_{\mathrm{A}}(x) \Delta_{\mathrm{A}} \Delta_{4}^{*} \chi_{\mathrm{A}}(x)\right)\right],
$$

with the matrices $P_{\mu}^{\prime}$, whose explicit form is given in appendix C.2, satisfying

$$
\begin{aligned}
& \left\{\gamma_{\mu}, \gamma_{\nu}\right\}=-2 \delta_{\mu \nu}, \quad\left\{P_{i}^{\prime}, P_{j}^{\prime}\right\}=2 \delta_{i j}, \quad\left\{\gamma_{i}, P_{j}^{\prime}\right\}=0 \\
& \left\{P_{4}^{\prime}, P_{i}^{\prime}\right\} \neq 0, \quad\left\{\gamma_{4}, P_{i}^{\prime}\right\} \neq 0, \quad\left\{\gamma_{i}, P_{4}^{\prime}\right\} \neq 0, \quad\left(P_{4}^{\prime}\right)^{2}=1 \quad(i, j=1,2,3) .
\end{aligned}
$$

Note that $\gamma_{i}$ anticommute with $P_{j}^{\prime}(i, j=1,2,3)$. From the same reason as $(2.33)$ in the case [2], the fermion kinetic term (2.47) has the unique zero-mode at $q_{1}=q_{2}=q_{3}=0$, after the dimensional reduction with respect to the fourth direction $x_{4}$ which kills the terms containing $\Delta_{4}$ or $\Delta_{4}^{*}$.

The four-dimensional modified action still has the surplus modes. However, for each of them the momentum has the nonzero fourth component $\left(q_{4} \neq 0\right)$, and they are removed in the models obtained by the dimensional reduction with respect to the fourth direction. In this way, we can define $\mathcal{N}=4$ lattice SYM models in two and three dimensions without surplus modes. We will see the details about the dimensionally reduced lattice models in the next section.

\section{Dimensionally Reduced $\mathcal{N}=4$ Models in Two and Three Dimensions}

We discuss on two- and three-dimensional $\mathcal{N}=4 \mathrm{SYM}$ models obtained by dimensional reduction from the slightly modified $\mathcal{N}=2$ model in four dimensions.

\section{$3.13 \mathrm{D} \mathcal{N}=4$ Case}

Here, we consider the dimensional reduction with respect to $x_{4}$ in the slightly modified model in section 2.2. The result leads $\mathcal{N}=4 \mathrm{SYM}$ on the three-dimensional lattice, whose action $S_{3 D \mathcal{N}=4}^{\mathrm{LAT}}+\Delta S^{\prime}$ is expressed as

$$
\begin{aligned}
S_{3 D \mathcal{N}=4}^{\mathrm{LAT}}=Q \frac{1}{2 g_{0}^{2}} \sum_{x} \operatorname{tr} & {\left[\frac{1}{4} \eta(x)[\phi(x), \bar{\phi}(x)]-i \vec{\chi}(x) \cdot\left(\vec{\Phi}^{\prime}(x)+\Delta \vec{\Phi}^{\prime}(x)\right)+\vec{\chi}(x) \cdot \vec{H}(x)\right.} \\
+ & i \sum_{\mu=1}^{3} \psi_{\mu}(x)\left(\bar{\phi}(x)-U_{\mu}(x) \bar{\phi}(x+\hat{\mu}) U_{\mu}(x)^{\dagger}\right) \\
& \left.+i \psi_{4}(x)\left(\bar{\phi}(x)-V(x) \bar{\phi}(x) V(x)^{\dagger}\right)\right]
\end{aligned}
$$

where $x$ represents the three-dimensional lattice site $\left(x_{1}, x_{2}, x_{3}\right)$, and $V(x) \equiv U_{4}(x)$, $V(x)^{\dagger}=U_{-4}(x)$ become sitting at the site $x . \Delta S^{\prime}$ is given by the formula (2.26) with the replacement $\vec{\Phi}(x) \rightarrow \vec{\Phi}^{\prime}(x), \Delta \vec{\Phi}(x) \rightarrow \Delta \vec{\Phi}^{\prime}(x)$ :

$$
\Delta S^{\prime}=\frac{1}{8 N g_{0}^{2}} \sum_{x}\left[\operatorname{tr}\left(\vec{\Phi}^{\prime}(x)+\Delta \vec{\Phi}^{\prime}(x)\right)\right]^{2} .
$$

Also, plaquettes $U_{-\mu, 4}(x)$ collapse to the links:

$$
U_{-\mu, 4}(x)=U_{\mu}(x-\hat{\mu})^{\dagger} V(x-\hat{\mu}) U_{\mu}(x-\hat{\mu}) V(x)^{\dagger}=U_{4,-\mu}(x)^{\dagger} \quad(\mu=1,2,3) .
$$


The manifest supersymmetry is given by the dimensional reduction of (2.6). Namely, for the cases $\mu=1,2,3$ of (2.6), the form does not change, while for the $\mu=4$ case it becomes

$$
Q V(x)=i \psi_{4}(x) V(x), \quad Q \psi_{4}(x)=i \psi_{4}(x) \psi_{4}(x)-i\left(\phi(x)-V(x) \phi(x) V(x)^{\dagger}\right) .
$$

The action $S_{3 D \mathcal{N}=4}^{\mathrm{LAT}}$ is explicitly written as

$$
\begin{aligned}
S_{3 D \mathcal{N}=4}^{\mathrm{LAT}}= & \frac{1}{2 g_{0}^{2}} \sum_{x} \operatorname{tr}\left[\frac{1}{4}[\phi(x), \bar{\phi}(x)]^{2}+\vec{H}(x) \cdot \vec{H}(x)-i \vec{H}(x) \cdot\left(\vec{\Phi}^{\prime}(x)+\Delta \vec{\Phi}^{\prime}(x)\right)\right. \\
& +\sum_{\mu=1}^{3}\left(\phi(x)-U_{\mu}(x) \phi(x+\hat{\mu}) U_{\mu}(x)^{\dagger}\right)\left(\bar{\phi}(x)-U_{\mu}(x) \bar{\phi}(x+\hat{\mu}) U_{\mu}(x)^{\dagger}\right) \\
& +\left(\phi(x)-V(x) \phi(x) V(x)^{\dagger}\right)\left(\bar{\phi}(x)-V(x) \bar{\phi}(x) V(x)^{\dagger}\right) \\
& -\frac{1}{4} \eta(x)[\phi(x), \eta(x)]-\vec{\chi}(x) \cdot[\phi(x), \vec{\chi}(x)] \\
& -\sum_{\mu=1}^{3} \psi_{\mu}(x) \psi_{\mu}(x)\left(\bar{\phi}(x)+U_{\mu}(x) \bar{\phi}(x+\hat{\mu}) U_{\mu}(x)^{\dagger}\right) \\
& -\psi_{4}(x) \psi_{4}(x)\left(\bar{\phi}(x)+V(x) \bar{\phi}(x) V(x)^{\dagger}\right)+i \vec{\chi}(x) \cdot Q\left(\vec{\Phi}^{\prime}(x)+\Delta \vec{\Phi}^{\prime}(x)\right) \\
& -i \sum_{\mu=1}^{3} \psi_{\mu}(x)\left(\eta(x)-U_{\mu}(x) \eta(x+\hat{\mu}) U_{\mu}(x)^{\dagger}\right) \\
& \left.-i \psi_{4}(x)\left(\eta(x)-V(x) \eta(x) V(x)^{\dagger}\right)\right] .
\end{aligned}
$$

Integrating out the auxiliary fields $\vec{H}(x)$ and combining the result with $\Delta S$ leads the term $\frac{1}{8 g_{0}^{2}} \sum_{x} \operatorname{tr}\left[\vec{\Phi}^{\prime}(x)+\Delta \vec{\Phi}^{\prime}(x)\right]^{2}$. According to the result of appendix $\mathrm{B}$, the minimum $\overrightarrow{\Phi^{\prime}}(x)+\Delta \vec{\Phi}^{\prime}(x)=0$ is given by the configurations satisfying

$$
\begin{aligned}
& U_{23}(x)=U_{31}(x)=U_{12}(x)=1, \\
& U_{4,-1}(x)=U_{4,-2}(x)=U_{4,-3}(x)=1 .
\end{aligned}
$$

On the infinite lattice $x \in \mathbf{Z}^{3}$, eqs. (3.6) are equivalent to $U_{1}(x)=U_{2}(x)=U_{3}(x)=1$ up to gauge transformations, and then eqs. (3.7) mean $V(x)=V_{0}$ ( $x$-independent constant). Furthermore, minimizing the term

$$
\operatorname{tr}\left(\phi(x)-V(x) \phi(x) V(x)^{\dagger}\right)\left(\bar{\phi}(x)-V(x) \bar{\phi}(x) V(x)^{\dagger}\right)
$$

in the action for arbitrary $\phi(x), \bar{\phi}(x)$ requires $V_{0} \in \mathbf{Z}_{N}$, i.e. a center element of $\mathrm{SU}(N)$. Since all the $\mathbf{Z}_{N}$ elements for $V(x)=V_{0}$ give the identical value to the action, it is sufficient to consider the expansion of $V(x)$ around a representative element of $\mathbf{Z}_{N}$, say $1_{N}$. (If all the elements are taken into account, we end up merely with the overall factor $N$ multiplied to the partition function, which gives no influence on physics of the system.) Thus, we can justify the weak field expansion

$$
U_{\mu}(x)=e^{i a A_{\mu}(x)}=1+i a A_{\mu}(x)+\cdots, \quad V(x)=e^{i a v(x)}=1+i a v(x)+\cdots .
$$


About Surplus Modes As argued in section 2.2, the fermion kinetic term in this threedimensional model has no surplus mode, which obviously means fermion doublers not appearing. Then the exact supersymmetry $Q$ guarantees nonexistence of the surplus modes in the bosonic sector. Now we explicitly check it. After the weak field expansion (3.9), the Fourier transformation of $\vec{\Phi}^{\prime}(x)$ becomes

$$
\begin{aligned}
& \tilde{\Phi}_{1}^{\prime}(q)=2 a\left[\left(1-e^{-i a q_{1}}\right) \tilde{v}(q)+\left(e^{i a q_{2}}-1\right) \tilde{A}_{3}(q)-\left(e^{i a q_{3}}-1\right) \tilde{A}_{2}(q)\right]+O\left(\tilde{v}^{2}, \tilde{A}^{2}\right), \\
& \tilde{\Phi}_{2}^{\prime}(q)=2 a\left[\left(1-e^{-i a q_{2}}\right) \tilde{v}(q)+\left(e^{i a q_{3}}-1\right) \tilde{A}_{1}(q)-\left(e^{i a q_{1}}-1\right) \tilde{A}_{3}(q)\right]+O\left(\tilde{v}^{2}, \tilde{A}^{2}\right), \\
& \tilde{\Phi}_{3}^{\prime}(q)=2 a\left[\left(1-e^{-i a q_{3}}\right) \tilde{v}(q)+\left(e^{i a q_{1}}-1\right) \tilde{A}_{2}(q)-\left(e^{i a q_{2}}-1\right) \tilde{A}_{1}(q)\right]+O\left(\tilde{v}^{2}, \tilde{A}^{2}\right) \cdot(3.1
\end{aligned}
$$

We do not have to take into account $\Delta \vec{\Phi}^{\prime}(x)$, because its weak field expansion merely yields irrelevant interaction terms and does not contribute to the kinetic term.

We can show that there is no nontrivial mode satisfying

$$
\tilde{\Phi}_{1}^{\prime}(q)=\tilde{\Phi}_{2}^{\prime}(q)=\tilde{\Phi}_{3}^{\prime}(q)=0+O\left(\tilde{v}^{2}, \tilde{A}^{2}\right)
$$

except $q_{1}=q_{2}=q_{3}=0$. First, let us consider the cases that the momentum $\left(q_{1}, q_{2}, q_{3}\right)$ has only one nonzero component. Since eqs. (3.11) are invariant under the simultaneous permutations $\left(q_{1}, q_{2}, q_{3}\right) \rightarrow\left(q_{2}, q_{3}, q_{1}\right)$ and $\left(\tilde{A}_{1}, \tilde{A}_{2}, \tilde{A}_{3}\right) \rightarrow\left(\tilde{A}_{2}, \tilde{A}_{3}, \tilde{A}_{1}\right)$, it is sufficient to see the case $q_{1}=q_{2}=0, q_{3} \neq 0$, alone. Then, eqs. (3.11) lead $\tilde{v}=\tilde{A}_{1}=\tilde{A}_{2}=0$, which can be written as

$$
\tilde{v}^{\alpha}(q)=0, \quad \tilde{A}_{\mu}^{\alpha}(q)=\left(e^{i a q_{\mu}}-1\right) K^{\alpha}(q)
$$

where $\alpha$ represents the index with respect to the $\mathrm{SU}(N)$ gauge generators, and $K^{\alpha}(q)$ are complex-valued functions of $q=\left(0,0, q_{3}\right)$. It is not physically nontrivial, because it is a longitudinal mode and can be absorbed by the gauge degrees of freedom.

Also, for the cases that the momentum has two nonzero components, the solution is described as the form (3.12) again and it is not physically nontrivial. Finally, in the case of all the components of the momentum nonzero, under the gauge choice of $\tilde{A}_{1}(q)=0$ one can write $\overrightarrow{\tilde{\Phi}^{\prime}}$ as

$$
\overrightarrow{\tilde{\Phi}^{\prime}}(q)=M(q)\left(\begin{array}{c}
\tilde{v}(q) \\
\tilde{A}_{2}(q) \\
\tilde{A}_{3}(q)
\end{array}\right)
$$

with

$$
M(q) \equiv\left[\begin{array}{ccc}
1-e^{-i a q_{1}} & 1-e^{i a q_{3}} & e^{i a q_{2}}-1 \\
1-e^{-i a q_{2}} & 0 & 1-e^{i a q_{1}} \\
1-e^{-i a q_{3}} & e^{i a q_{1}}-1 & 0
\end{array}\right]
$$

The determinant of the matrix $M(q)$ takes the form

$$
\operatorname{det} M(q)=-4\left(e^{i a q_{1}}-1\right)\left[\sin ^{2}\left(\frac{a q_{1}}{2}\right)+\sin ^{2}\left(\frac{a q_{2}}{2}\right)+\sin ^{2}\left(\frac{a q_{3}}{2}\right)\right] .
$$

Since $\operatorname{det} M(q) \neq 0$ for $q_{1}, q_{2}, q_{3} \neq 0$, there is no nontrivial solution for (3.11).

Thus, the boson kinetic term has no physical surplus modes, which precisely coincides with the situation for the fermionic sector as expected by the existence of the exact supersymmetry $Q$. 
Renormalization After the rescaling

$$
\begin{aligned}
& \phi(x) \rightarrow a \phi(x), \quad \bar{\phi}(x) \rightarrow a \bar{\phi}(x), \quad \vec{H}(x) \rightarrow a^{2} \vec{H}(x), \quad \psi_{\mu}(x) \rightarrow a^{3 / 2} \psi_{\mu}(x), \\
& \psi_{4}(x) \rightarrow a^{3 / 2} \psi_{4}(x), \quad \vec{\chi}(x) \rightarrow a^{3 / 2} \vec{\chi}(x), \quad \eta(x) \rightarrow a^{3 / 2} \eta(x),
\end{aligned}
$$

the lattice action (3.1) reduces to the continuum action of $\mathcal{N}=4 \mathrm{SYM}$ in the continuum limit $a \rightarrow 0$ with $g_{3}^{2} \equiv g_{0}^{2} / a$ fixed, and thus $\mathcal{N}=4$ supersymmetry and rotational symmetry in three dimensions are restored in the classical sense. The term $\Delta S^{\prime}$ is of order $O\left(a^{4}\right)$ and becomes irrelevant. We will check whether the symmetry restoration persists against the quantum corrections, i.e. whether symmetries of the lattice action forbid relevant or marginal operators induced which possibly obstruct the symmetry restoration. In order to consider the quantum effects near the continuum limit, we assume the fixed point at $g_{0}=0$, which is suggested by the asymptotic freedom of the theory, and treat quantum fluctuations in the perturbative way around $g_{0}=0$.

First let us consider the action $S_{3 D \mathcal{N}=4}^{\mathrm{LAT}}$ without the term $\Delta S^{\prime}$. It is useful for the renormalization argument to note symmetries of the lattice action (3.1):

- lattice translational symmetry

- $\mathrm{SU}(N)$ gauge symmetry

- supersymmetry $Q$

- global $\mathrm{U}(1)_{R}$ internal symmetry ${ }^{4}$ of the same form as (2.7)

- cyclic permutation $x \equiv\left(x_{1}, x_{2}, x_{3}\right) \rightarrow \tilde{x} \equiv\left(x_{3}, x_{1}, x_{2}\right)$ as

$$
\begin{aligned}
\left(U_{1}(x), U_{2}(x), U_{3}(x), V(x)\right) & \rightarrow\left(U_{2}(\tilde{x}), U_{3}(\tilde{x}), U_{1}(\tilde{x}), V(\tilde{x})\right) \\
\left(\psi_{1}(x), \psi_{2}(x), \psi_{3}(x), \psi_{4}(x)\right) & \rightarrow\left(\psi_{2}(\tilde{x}), \psi_{3}(\tilde{x}), \psi_{1}(\tilde{x}), \psi_{4}(\tilde{x})\right) \\
\left(H_{1}(x), H_{2}(x), H_{3}(x)\right) & \rightarrow\left(H_{2}(\tilde{x}), H_{3}(\tilde{x}), H_{1}(\tilde{x})\right) \\
\left(\chi_{1}(x), \chi_{2}(x), \chi_{3}(x)\right) & \rightarrow\left(\chi_{2}(\tilde{x}), \chi_{3}(\tilde{x}), \chi_{1}(\tilde{x})\right) \\
(\phi(x), \bar{\phi}(x)) & \rightarrow(\phi(\tilde{x}), \bar{\phi}(\tilde{x})) \\
\eta(x) & \rightarrow \eta(\tilde{x}) .
\end{aligned}
$$

As for the cyclic permutation, $x \pm \hat{1}, x \pm \hat{2}, x \pm \hat{3}$ are transformed to $\tilde{x} \pm \hat{2}, \tilde{x} \pm \hat{3}, \tilde{x} \pm \hat{1}$, respectively. Then, one can see that

$$
\begin{aligned}
& \Phi_{1}^{\prime}(x)+\Delta \Phi_{1}^{\prime}(x) \rightarrow \Phi_{2}^{\prime}(\tilde{x})+\Delta \Phi_{2}^{\prime}(\tilde{x}), \\
& \Phi_{2}^{\prime}(x)+\Delta \Phi_{2}^{\prime}(x) \rightarrow \Phi_{3}^{\prime}(\tilde{x})+\Delta \Phi_{3}^{\prime}(\tilde{x}), \\
& \Phi_{3}^{\prime}(x)+\Delta \Phi_{3}^{\prime}(x) \rightarrow \Phi_{1}^{\prime}(\tilde{x})+\Delta \Phi_{1}^{\prime}(\tilde{x}),
\end{aligned}
$$

and that the action is invariant under (3.17).

\footnotetext{
${ }^{4}$ Because the theory is defined on odd-dimensional space-time, the $\mathrm{U}(1)_{R}$ symmetry does not become anomalous.
} 


\begin{tabular}{|c|c|c|c|c|c|}
\hline $\bar{p} p=a+b+3 c$ & \multicolumn{5}{|c|}{$\overline{\varphi^{a} \partial^{b} \psi^{2 c}}$} \\
\hline 0 & \multicolumn{5}{|c|}{1} \\
\hline 1 & \multicolumn{5}{|c|}{$\varphi$} \\
\hline 2 & & & $\varphi^{2}$ & & \\
\hline 3 & & $\varphi^{3}$, & $\psi \psi$ & $\varphi \partial \varphi$ & \\
\hline 4 & $\varphi^{4}$, & $\varphi^{2} \partial \varphi$ & $(\partial \varphi)^{2}$ & $\psi \partial \psi$ & $\varphi \psi \psi$ \\
\hline
\end{tabular}

Table 1: List of operators with $p \leq 4$.

The mass dimension of the coupling constant squared $g_{3}^{2}$ is one. For a generic boson field $\varphi$ (except the auxiliary fields $\vec{H}$ ) and a fermion field $\psi$, the dimensions are 1 and $3 / 2$ respectively. Thus, operators of the type $\varphi^{a} \partial^{b} \psi^{2 c}$ have the dimension $p \equiv a+b+3 c$, where ' $\partial$ ' means the derivative with respect to a coordinate. From the dimensional analysis, it turns out that the operators receive the following radiative corrections up to some powers of possible logarithmic factors:

$$
\left(\frac{a^{p-4}}{g_{3}^{2}}+c_{1} a^{p-3}+c_{2} a^{p-2} g_{3}^{2}+c_{3} a^{p-1} g_{3}^{4}+c_{4} a^{p} g_{3}^{6}+\cdots\right) \int \mathrm{d}^{3} x \varphi^{a} \partial^{b} \psi^{2 c},
$$

where the first term in the parentheses represents the contribution from the tree level, and the term containing the coefficient $c_{\ell}$ comes from the $\ell$-loop contribution. It is clear from $g_{3}^{2}$ playing the same role as the Planck constant $\hbar$ in the action (3.1). From the formula (3.19), one can see that operators with $p \leq 4-\ell$ are relevant or marginal if they are induced at the $\ell$-loop level.

Because we know that the lattice action reduces to the desired continuum SYM action at the classical level, we need to check operators with $p=0,1,2,3$ which are allowed to be induced by the above symmetries of the lattice action. Operators with $p \leq 4$ are listed in Table 1. The identity operator corresponding to $p=0$ merely shifts the origin of the energy, which is not interesting to us. For the cases $p=1,2$, the $\mathrm{SU}(N)$ gauge invariance and the $\mathrm{U}(1)_{R}$ symmetry allow the scalar mass operators $\operatorname{tr}(\phi \bar{\phi}), \operatorname{tr} v^{2}$, while they are forbidden by the supersymmetry $Q$.

In the $p=3$ case, let us first consider operators of the $Q$-exact form: $Q \mathcal{O}$ as $Q$-invariant operators. Then $\mathcal{O}$ has the mass dimension $5 / 2$ and the $\mathrm{U}(1)_{R}$ charge -1 . Candidates of such $\mathcal{O}$ are $\operatorname{tr}\left(\psi_{\mu} \bar{\phi}\right), \operatorname{tr}\left(\psi_{4} \bar{\phi}\right), \operatorname{tr}\left(\chi_{\mathbf{A}} v\right)$. Here, we do not have to take into account $\operatorname{tr}(\eta v)$, because $Q \operatorname{tr}(\eta v)=-Q \operatorname{tr}\left(\psi_{4} \bar{\phi}\right)$. Due to the requirement of the permutation symmetry (3.17), the candidates reduce to the following three combinations:

$$
\sum_{\mu=1}^{3} \operatorname{tr}\left(\psi_{\mu} \bar{\phi}\right), \quad \operatorname{tr}\left(\psi_{4} \bar{\phi}\right), \quad \sum_{\mathrm{A}=1}^{3} \operatorname{tr}\left(\chi_{\mathbf{A}} v\right)
$$

The corresponding $p=3$ operators are

$$
Q \sum_{\mu=1}^{3} \operatorname{tr}\left(\psi_{\mu} \bar{\phi}\right)=\sum_{\mu=1}^{3} \operatorname{tr}\left(i \bar{\phi} D_{\mu} \phi-\psi_{\mu} \eta\right), \quad Q \operatorname{tr}\left(\psi_{4} \bar{\phi}\right)=\operatorname{tr}\left(-[\phi, \bar{\phi}] v+\eta \psi_{4}\right),
$$




$$
Q \sum_{\mathrm{A}=1}^{3} \operatorname{tr}\left(\chi_{\mathrm{A}} v\right)=\sum_{\mathrm{A}=1}^{3} \operatorname{tr}\left(H_{\mathrm{A}} v-\chi_{\mathrm{A}} \psi_{4}\right)
$$

Note that other than the fermion bilinears appearing in the three operators of (3.21) there are no possible $\psi \psi$ terms fulfilling the symmetry requirements. $\left(\operatorname{tr}\left(\psi_{\mu} \chi_{\mathrm{A}}\right)\right.$ comes from $Q \operatorname{tr}\left(A_{\mu} \chi_{\mathrm{A}}\right)$, while it is not gauge invariant.) Because any $p=3$ operators consistent to the symmetries must contain $\psi \psi$ terms, we can say that all the $p=3$ operators satisfying the symmetries are given by (3.21) and nothing more appears. It means that there is no nontrivial $Q$-cohomology element satisfying the symmetries of the lattice action, and we do not have to consider operators belonging to the nontrivial $Q$-cohomology. Thus, we can conclude that the three of (3.21) are the operators of $p=3$ possibly radiatively generated.

When taking into account the effect of the term $\Delta S^{\prime}$, it appears via the vertices of $\Delta S^{\prime}$ in the loop expansion. Since $\Delta S^{\prime}$ is of the order $O\left(a^{4}\right)$, it is easy to see that the effect to (3.19) becomes irrelevant in the continuum limit. Thus, the conclusion of the renormalization given above is valid without any change, even when the effect of $\Delta S^{\prime}$ is included.

Therefore, the loop corrections are allowed to generate the three operators (3.21) alone except the identity. In order to reach the desired supersymmetric continuum theory, we have to add counter terms for the operators and cancel them with the radiative corrections by fine-tuning.

\section{$3.22 \mathrm{D} \mathcal{N}=4$ Case}

A lattice model for $\mathcal{N}=4 \mathrm{SYM}$ in two dimensions is obtained as a result of the further dimensional reduction of (3.1) with respect to $x_{3}$. In addition to the variable $V(x)$ sitting on the two-dimensional lattice site $x=\left(x_{1}, x_{2}\right)$, there appear new site variables $W(x) \equiv U_{3}(x)$, $W(x)^{\dagger}=U_{-3}(x)$. The action $S_{2 D \mathcal{N}=4}^{\mathrm{LAT}}+\Delta S^{\prime}$ takes the form

$$
\begin{aligned}
S_{2 D \mathcal{N}}^{\mathrm{LAT}}= & =Q \frac{1}{2 g_{0}^{2}} \sum_{x} \operatorname{tr}\left[\frac{1}{4} \eta(x)[\phi(x), \bar{\phi}(x)]-i \vec{\chi}(x) \cdot\left(\vec{\Phi}^{\prime}(x)+\Delta \vec{\Phi}^{\prime}(x)\right)+\vec{\chi}(x) \cdot \vec{H}(x)\right. \\
& +i \sum_{\mu=1}^{2} \psi_{\mu}(x)\left(\bar{\phi}(x)-U_{\mu}(x) \bar{\phi}(x+\hat{\mu}) U_{\mu}(x)^{\dagger}\right) \\
& \left.+i \psi_{3}(x)\left(\bar{\phi}(x)-W(x) \bar{\phi}(x) W(x)^{\dagger}\right)+i \psi_{4}(x)\left(\bar{\phi}(x)-V(x) \bar{\phi}(x) V(x)^{\dagger}\right)\right]
\end{aligned}
$$

where $\overrightarrow{\Phi^{\prime}}, \Delta \overrightarrow{\Phi^{\prime}}$ are given by (2.44) and

$$
\begin{aligned}
& U_{-\mu, 4}(x)=U_{\mu}(x-\hat{\mu})^{\dagger} V(x-\hat{\mu}) U_{\mu}(x-\hat{\mu}) V(x)^{\dagger}=U_{4,-\mu}(x)^{\dagger}, \\
& U_{\mu 3}(x)=U_{\mu}(x) W(x+\hat{\mu}) U_{-\mu}(x+\hat{\mu}) W(x)^{\dagger}=U_{3 \mu}(x)^{\dagger}, \\
& U_{-3,4}(x)=W(x)^{\dagger} V(x) W(x) V(x)^{\dagger}=U_{4,-3}(x)^{\dagger} \quad(\mu=1,2) .
\end{aligned}
$$

$\Delta S^{\prime}$ is given by the same expression as in (3.2).

The explicit form of the action is

$$
S_{2 D \mathcal{N}=4}^{\mathrm{LAT}}=\frac{1}{2 g_{0}^{2}} \sum_{x} \operatorname{tr}\left[\frac{1}{4}[\phi(x), \bar{\phi}(x)]^{2}+\vec{H}(x) \cdot \vec{H}(x)-i \vec{H}(x) \cdot\left(\vec{\Phi}^{\prime}(x)+\Delta \vec{\Phi}^{\prime}(x)\right)\right.
$$




$$
\begin{aligned}
& +\sum_{\mu=1}^{2}\left(\phi(x)-U_{\mu}(x) \phi(x+\hat{\mu}) U_{\mu}(x)^{\dagger}\right)\left(\bar{\phi}(x)-U_{\mu}(x) \bar{\phi}(x+\hat{\mu}) U_{\mu}(x)^{\dagger}\right) \\
& +\left(\phi(x)-W(x) \phi(x) W(x)^{\dagger}\right)\left(\bar{\phi}(x)-W(x) \bar{\phi}(x) W(x)^{\dagger}\right) \\
& +\left(\phi(x)-V(x) \phi(x) V(x)^{\dagger}\right)\left(\bar{\phi}(x)-V(x) \bar{\phi}(x) V(x)^{\dagger}\right) \\
& -\frac{1}{4} \eta(x)[\phi(x), \eta(x)]-\vec{\chi}(x) \cdot[\phi(x), \vec{\chi}(x)] \\
& -\sum_{\mu=1}^{2} \psi_{\mu}(x) \psi_{\mu}(x)\left(\bar{\phi}(x)+U_{\mu}(x) \bar{\phi}(x+\hat{\mu}) U_{\mu}(x)^{\dagger}\right) \\
& -\psi_{3}(x) \psi_{3}(x)\left(\bar{\phi}(x)+W(x) \bar{\phi}(x) W(x)^{\dagger}\right)-\psi_{4}(x) \psi_{4}(x)\left(\bar{\phi}(x)+V(x) \bar{\phi}(x) V(x)^{\dagger}\right) \\
& +i \vec{\chi}(x) \cdot Q\left(\vec{\Phi}^{\prime}(x)+\Delta \vec{\Phi}^{\prime}(x)\right)-i \sum_{\mu=1}^{2} \psi_{\mu}(x)\left(\eta(x)-U_{\mu}(x) \eta(x+\hat{\mu}) U_{\mu}(x)^{\dagger}\right) \\
& \left.-i \psi_{3}(x)\left(\eta(x)-W(x) \eta(x) W(x)^{\dagger}\right)-i \psi_{4}(x)\left(\eta(x)-V(x) \eta(x) V(x)^{\dagger}\right)\right] .
\end{aligned}
$$

From the argument parallel to the three-dimensional $\mathcal{N}=4$ case, the action is minimized by the configurations gauge-equivalent to $U_{\mu}(x)=1, V(x)=V_{0} \in \mathbf{Z}_{N}, W(x)=W_{0} \in \mathbf{Z}_{N}$. Since all the elements of $\mathbf{Z}_{N}$ for $V_{0}$ and $W_{0}$ give the same value to the action, it is justified to consider the weak field expansion around 1 as

$$
\begin{aligned}
& U_{\mu}(x)=e^{i a A_{\mu}(x)}=1+i a A_{\mu}(x)+\cdots, \quad V(x)=e^{i a v(x)}=1+i a v(x)+\cdots, \\
& W(x)=e^{i a w(x)}=1+i a w(x)+\cdots .
\end{aligned}
$$

It reproduces the continuum action of the theory having the full $\mathcal{N}=4$ supersymmetry in the classical continuum limit: $a \rightarrow 0$ with the coupling constant squared $g_{2}^{2}=g_{0}^{2} / a^{2}$ fixed.

Because the three-dimensional $\mathcal{N}=4 \mathrm{SYM}$ model in the previous subsection does not have any surplus modes, obviously no surplus mode appears in the two-dimensional $\mathcal{N}=4$ case here obtained by the further dimensional reduction therefrom ${ }^{5}$.

The argument for the renormalization goes along similarly to the three-dimensional $\mathcal{N}=4$ case. Now the coupling squared $g_{2}^{2}$ has the mass dimension two, and generic operators of the type $\varphi^{a} \partial^{b} \psi^{2 c}$ with the dimension $p \equiv a+b+3 c$ receive the loop corrections as

$$
\left(\frac{a^{p-4}}{g_{2}^{2}}+c_{1} a^{p-2}+c_{2} a^{p} g_{2}^{2}+\cdots\right) \int \mathrm{d}^{2} x \varphi^{a} \partial^{b} \psi^{2 c},
$$

where the notations are similar as in (3.19). $\Delta S^{\prime}$ is of the order $O\left(a^{4}\right)$, and becomes irrelevant in the renormalization argument. To seek possible relevant or marginal operators radiatively generated, let us see operators of $p=1,2$, which are of the types $\varphi$ and $\varphi^{2}$ according to Table 1. Among them, there does not exist any operator satisfying the $\mathrm{SU}(N)$ gauge invariance, the $\mathrm{U}(1)_{R}$ symmetry and the supersymmetry $Q$. Thus, the loop corrections are not allowed to generate any relevant or marginal operators except the identity,

\footnotetext{
${ }^{5}$ In fact, the fermion kinetic part of (3.24) takes the same form to the model based on the 'BTFT formulation' preserving the two supercharges given in ref. [2]. The latter clearly has no surplus modes because of the property (2.33). It also proves the nonexistence of the surplus modes for (3.24).
} 
which means restoration of the full $\mathcal{N}=4$ supersymmetry and rotational invariance in the continuum limit without any fine-tuning.

The lattice action (3.22) preserving one supercharge $Q$ is different from the formulation based on the BTFT discussed in [2], where two supercharges $Q_{ \pm}$are maintained. Thus, we have two different lattice formulations describing the same target theory of two-dimensional $\mathcal{N}=4$ SYM with no tuning of parameters.

\section{Naive $4 \mathrm{D} \mathcal{N}=4$ Lattice Model and Its Slight Modification}

Here, we discuss on a naive lattice model for four-dimensional $\mathcal{N}=4 \mathrm{SYM}$ as well as its slightly modified version. Notations are same as in ref. [1].

\subsection{Naive Lattice Model for $4 \mathrm{D} \mathcal{N}=4$}

Let us start from the naive lattice action for four-dimensional $\mathcal{N}=4 \mathrm{SYM}$ theory with two supercharges $Q_{ \pm}$preserved:

$$
\begin{aligned}
S_{4 D \mathcal{N}=4}^{\mathrm{LAT}}=Q_{+} Q_{-} \frac{1}{2 g_{0}^{2}} \sum_{x} \operatorname{tr}[-i \vec{B}(x) \cdot(\vec{\Phi}(x)+\Delta \vec{\Phi}(x)) \\
-\frac{1}{3} \sum_{\mathrm{A}, \mathrm{B}, \mathrm{C}=1}^{3} \varepsilon_{\mathrm{ABC}} B_{\mathrm{A}}(x)\left[B_{\mathrm{B}}(x), B_{\mathrm{C}}(x)\right] \\
\left.-\sum_{\mu=1}^{4} \psi_{+\mu}(x) \psi_{-\mu}(x)-\vec{\chi}_{+}(x) \cdot \vec{\chi}_{-}(x)-\frac{1}{4} \eta_{+}(x) \eta_{-}(x)\right]
\end{aligned}
$$

where quantities with the arrows are three-component vectors, and $\vec{\Phi}(x), \Delta \vec{\Phi}(x)$ are given by (2.3). In the $\mathcal{N}=4 \mathrm{SYM}$, we have real scalar fields $\vec{B}, C$ (hermitian matrices) in addition to the complex scalars $\phi, \bar{\phi}$. Also, $\tilde{H}_{\mu}, \vec{H}$ are bosonic auxiliary fields. Fields $\psi_{+\mu}$, $\psi_{-\mu}, \vec{\chi}_{+}, \vec{\chi}_{-}, \eta_{+}, \eta_{-}$represent fermions. The $Q_{ \pm}$supersymmetry transforms the lattice fields as

$$
\begin{aligned}
Q_{+} U_{\mu}(x)= & i \psi_{+\mu}(x) U_{\mu}(x), \\
Q_{-} U_{\mu}(x)= & i \psi_{-\mu}(x) U_{\mu}(x) \\
Q_{+} \psi_{+\mu}(x)= & i \psi_{+\mu} \psi_{+\mu}(x)-i\left(\phi(x)-U_{\mu}(x) \phi(x+\hat{\mu}) U_{\mu}(x)^{\dagger}\right), \\
Q_{-} \psi_{-\mu}(x)= & i \psi_{-\mu} \psi_{-\mu}(x)+i\left(\bar{\phi}(x)-U_{\mu}(x) \bar{\phi}(x+\hat{\mu}) U_{\mu}(x)^{\dagger}\right), \\
Q_{-} \psi_{+\mu}(x)= & \frac{i}{2}\left\{\psi_{+\mu}(x), \psi_{-\mu}(x)\right\}-\frac{i}{2}\left(C(x)-U_{\mu}(x) C(x+\hat{\mu}) U_{\mu}(x)^{\dagger}\right)-\tilde{H}_{\mu}(x), \\
Q_{+} \psi_{-\mu}(x)= & \frac{i}{2}\left\{\psi_{+\mu}(x), \psi_{-\mu}(x)\right\}-\frac{i}{2}\left(C(x)-U_{\mu}(x) C(x+\hat{\mu}) U_{\mu}(x)^{\dagger}\right)+\tilde{H}_{\mu}(x), \\
Q_{+} \tilde{H}_{\mu}(x)= & -\frac{1}{2}\left[\psi_{-\mu}(x), \phi(x)+U_{\mu}(x) \phi(x+\hat{\mu}) U_{\mu}(x)^{\dagger}\right] \\
& +\frac{1}{4}\left[\psi_{+\mu}(x), C(x)+U_{\mu}(x) C(x+\hat{\mu}) U_{\mu}(x)^{\dagger}\right] \\
& +\frac{i}{2}\left(\eta_{+}(x)-U_{\mu}(x) \eta_{+}(x+\hat{\mu}) U_{\mu}(x)^{\dagger}\right)
\end{aligned}
$$




$$
\begin{aligned}
&+\frac{i}{2}\left[\psi_{+\mu}(x), \tilde{H}_{\mu}(x)\right]+\frac{1}{4}\left[\psi_{+\mu}(x) \psi_{+\mu}(x), \psi_{-\mu}(x)\right], \\
& Q_{-} \tilde{H}_{\mu}(x)=-\frac{1}{2}\left[\psi_{+\mu}(x), \bar{\phi}(x)+U_{\mu}(x) \bar{\phi}(x+\hat{\mu}) U_{\mu}(x)^{\dagger}\right] \\
&-\frac{1}{4}\left[\psi_{-\mu}(x), C(x)+U_{\mu}(x) C(x+\hat{\mu}) U_{\mu}(x)^{\dagger}\right] \\
&-\frac{i}{2}\left(\eta_{-}(x)-U_{\mu}(x) \eta_{-}(x+\hat{\mu}) U_{\mu}(x)^{\dagger}\right) \\
&+ \frac{i}{2}\left[\psi_{-\mu}(x), \tilde{H}_{\mu}(x)\right]-\frac{1}{4}\left[\psi_{-\mu}(x) \psi_{-\mu}(x), \psi_{+\mu}(x)\right], \\
& Q_{+} \vec{B}(x)=\vec{\chi}_{+}(x), \quad Q_{+} \vec{\chi}_{+}(x)=[\phi(x), \vec{B}(x)], \\
& Q_{-} \vec{B}(x)=\vec{\chi}_{-}(x), \quad Q_{-} \vec{\chi}_{-}(x)=-[\bar{\phi}(x), \vec{B}(x)], \\
& Q_{-} \chi_{+\mathrm{A}}(x)=\frac{1}{2}\left[C(x), B_{\mathrm{A}}(x)\right]-\frac{1}{2} \varepsilon_{\mathrm{ABC}}\left[B_{\mathrm{B}}(x), B_{\mathrm{C}}(x)\right]-H_{\mathrm{A}}(x), \\
& Q_{+} \chi_{-\mathrm{A}}(x)=\frac{1}{2}\left[C(x), B_{\mathrm{A}}(x)\right]+\frac{1}{2} \varepsilon_{\mathrm{ABC}}\left[B_{\mathrm{B}}(x), B_{\mathrm{C}}(x)\right]+H_{\mathrm{A}}(x), \\
& Q_{+} H_{\mathrm{A}}(x)=\left[\phi(x), \chi_{-\mathrm{A}}(x)\right]+\frac{1}{2}\left[B_{\mathrm{A}}(x), \eta_{+}(x)\right]-\frac{1}{2}\left[C(x), \chi_{+\mathrm{A}}(x)\right] \\
& \quad-\varepsilon_{\mathrm{ABC}}\left[B_{\mathrm{B}}(x), \chi_{+\mathrm{C}}(x)\right], \\
& Q_{-} H_{\mathrm{A}}(x)=\left[\bar{\phi}(x), \chi_{+\mathrm{A}}(x)\right]-\frac{1}{2}\left[B_{\mathrm{A}}(x), \eta_{-}(x)\right]+\frac{1}{2}\left[C(x), \chi_{-\mathrm{A}}(x)\right] \\
& \quad-\varepsilon_{\mathrm{ABC}}\left[B_{\mathrm{B}}(x), \chi_{-\mathrm{C}}(x)\right], \\
& Q_{+} C(x)=\eta_{+}(x), \quad Q_{+} \eta_{+}(x)=[\phi(x), C(x)], \quad Q_{-} \eta_{+}(x)=-[\phi(x), \bar{\phi}(x)], \\
&\left.Q_{-}=0, \quad Q_{-} \phi(x)=-\eta_{+}(x), \quad Q_{+} \bar{\phi}(x)=\eta_{-}(x), \quad Q_{-} \bar{\phi}(x)=0 ., \bar{\phi}(x)\right],
\end{aligned}
$$

The supercharges $Q_{ \pm}$are nilpotent up to gauge transformations in the sense

$$
\begin{aligned}
Q_{+}^{2} & =(\text { infinitesimal gauge transformation with the parameter } \phi), \\
Q_{-}^{2} & =(\text { infinitesimal gauge transformation with the parameter }-\bar{\phi}), \\
\left\{Q_{+}, Q_{-}\right\} & =(\text {infinitesimal gauge transformation with the parameter } C) .
\end{aligned}
$$

As discussed in ref. [1], the action has the "double $Q$-exact form" $\left(Q_{+} Q_{-}\right.$(something)) based on the 'BTFT formulation' [4] of $\mathcal{N}=4$ theories. The order of various quantities on the lattice are as follows:

$$
\begin{aligned}
& \vec{B}(x), C(x), \phi(x), \bar{\phi}(x)=O(a), \quad \tilde{H}_{\mu}(x), \vec{H}(x)=O\left(a^{2}\right) \\
& \psi_{ \pm \mu}(x), \vec{\chi}_{ \pm}(x), \eta_{ \pm}(x)=O\left(a^{3 / 2}\right), \quad Q_{ \pm}=O\left(a^{1 / 2}\right) .
\end{aligned}
$$

Note that the model has an $\mathrm{SU}(2)_{R}$ symmetry, whose generators are defined as

$$
J_{++}=\sum_{x, \alpha}\left[\sum_{\mu=1}^{4} \psi_{+\mu}^{\alpha}(x) \frac{\partial}{\partial \psi_{-\mu}^{\alpha}(x)}+\sum_{\mathrm{A}=1}^{3} \chi_{+\mathrm{A}}^{\alpha}(x) \frac{\partial}{\partial \chi_{-\mathrm{A}}^{\alpha}(x)}-\eta_{+}^{\alpha}(x) \frac{\partial}{\partial \eta_{-}^{\alpha}(x)}\right.
$$




$$
\begin{aligned}
&\left.+2 \phi^{\alpha}(x) \frac{\partial}{\partial C^{\alpha}(x)}-C^{\alpha}(x) \frac{\partial}{\partial \bar{\phi}^{\alpha}(x)}\right] \\
& J_{--}=\sum_{x, \alpha}\left[\sum_{\mu=1}^{4} \psi_{-\mu}^{\alpha}(x) \frac{\partial}{\partial \psi_{+\mu}^{\alpha}(x)}+\sum_{\mathrm{A}=1}^{3} \chi_{-\mathrm{A}}^{\alpha}(x) \frac{\partial}{\partial \chi_{+\mathrm{A}}^{\alpha}(x)}-\eta_{-}^{\alpha}(x) \frac{\partial}{\partial \eta_{+}^{\alpha}(x)}\right. \\
&\left.-2 \bar{\phi}^{\alpha}(x) \frac{\partial}{\partial C^{\alpha}(x)}+C^{\alpha}(x) \frac{\partial}{\partial \phi^{\alpha}(x)}\right] \\
& J_{0}=\sum_{x, \alpha}\left[\sum_{\mu=1}^{4} \psi_{+\mu}^{\alpha}(x) \frac{\partial}{\partial \psi_{+\mu}^{\alpha}(x)}-\sum_{\mu=1}^{4} \psi_{-\mu}^{\alpha}(x) \frac{\partial}{\partial \psi_{-\mu}^{\alpha}(x)}+\sum_{\mathrm{A}=1}^{3} \chi_{+\mathrm{A}}^{\alpha}(x) \frac{\partial}{\partial \chi_{+\mathrm{A}}^{\alpha}(x)}\right.-\sum_{\mathrm{A}=1}^{3} \chi_{-\mathrm{A}}^{\alpha}(x) \frac{\partial}{\partial \chi_{-\mathrm{A}}^{\alpha}(x)}+\eta_{+}^{\alpha}(x) \frac{\partial}{\partial \eta_{+}^{\alpha}(x)}-\eta_{-}^{\alpha}(x) \frac{\partial}{\partial \eta_{-}^{\alpha}(x)} \\
&\left.+2 \phi^{\alpha}(x) \frac{\partial}{\partial \phi^{\alpha}(x)}-2 \bar{\phi}^{\alpha}(x) \frac{\partial}{\partial \bar{\phi}^{\alpha}(x)}\right]
\end{aligned}
$$

with $\alpha=1, \cdots, N^{2}-1$ the index of the $\mathrm{SU}(N)$ gauge group generators, satisfying the algebra:

$$
\left[J_{0}, J_{++}\right]=2 J_{++}, \quad\left[J_{0}, J_{--}\right]=-2 J_{--}, \quad\left[J_{++}, J_{--}\right]=J_{0} .
$$

The $\mathrm{SU}(2)_{R}$ symmetry is a subgroup of a full $\mathrm{SU}(4)_{R}$ symmetry group in four-dimensional $\mathcal{N}=4$ SYM theory. $J_{0}$ is a generator of $\mathrm{U}(1)_{R}$ rotation, which is contained in the $\mathrm{SU}(2)_{R}$ as its Cartan subalgebra. $J_{++}\left(J_{--}\right)$raises (lowers) the $\mathrm{U}(1)_{R}$ charge by two-units. The subscript \pm of the fermions indicates the $\mathrm{U}(1)_{R}$ charge \pm 1 , and $Q_{ \pm}$raises/lowers the charge by one. Under the $\mathrm{SU}(2)_{R}$ rotation, each of $\left(\psi_{+\mu}^{\alpha}, \psi_{-\mu}^{\alpha}\right),\left(\chi_{+}^{\alpha}, \chi_{-}^{\alpha}\right),\left(\eta_{+}^{\alpha},-\eta_{-}^{\alpha}\right)$ and $\left(Q_{+}, Q_{-}\right)$ transforms as a doublet, and $\left(\phi^{\alpha}, C^{\alpha},-\bar{\phi}^{\alpha}\right)$ as a triplet.

Similarly to the case of the four-dimensional $\mathcal{N}=2$ model, we consider the action ${ }^{6}$ $S_{4 D \mathcal{N}=4}^{\mathrm{LAT}}+\Delta S$. The minimum $\vec{\Phi}(x)+\Delta \vec{\Phi}(x)=0$ is given uniquely by $U_{\mu \nu}(x)=1$ for the parameter $r$ of (2.12). Thus, the weak field expansion of $U_{\mu}(x)$ around 1 is justified again, but the same kind of surplus modes appear both in the boson and fermion kinetic terms. We will focus on constructing lower-dimensional lattice models for $\mathcal{N}=8 \mathrm{SYM}$ theories obtained by the dimensional reduction from a slightly modified version of the four-dimensional model.

\subsection{Slightly Modified Model for $4 \mathrm{D} \mathcal{N}=4$}

Let us consider a slight modification of the four-dimensional model by replacing $\vec{\Phi}, \Delta \vec{\Phi}$ with $\overrightarrow{\Phi^{\prime}}, \Delta \overrightarrow{\Phi^{\prime}}$ defined in (2.44). After rescaling the fermion fields by $a^{3 / 2}$, the fermion kinetic term becomes

$$
S_{f}^{(2)}=\frac{a^{4}}{2 g_{0}^{2}} \sum_{x} \operatorname{tr}\left[\sum_{\mu=1}^{4}\left\{-\frac{1}{2} \Psi(x)^{T} \gamma_{\mu}\left(\Delta_{\mu}+\Delta_{\mu}^{*}\right) \Psi(x)-a \frac{1}{2} \Psi(x)^{T} P_{\mu}^{\prime} \Delta_{\mu} \Delta_{\mu}^{*} \Psi(x)\right\}\right.
$$

\footnotetext{
${ }^{6}$ We can consider to extend $\vec{B}(x), \vec{\chi}_{ \pm}(x), \vec{H}(x)$ to hermitian matrices with nonvanishing trace parts. Then we have to soak up the would-be zero-modes of $\vec{B}^{(0)}(x), \vec{\chi}_{ \pm}^{(0)}(x)$. By a similar argument to the $\mathcal{N}=2$ case, the effect is shown to be equivalent to adding the term $\Delta S$ (2.26) to the action (4.1).
} 


$$
\begin{aligned}
& +i a \sum_{\mathrm{A}=1}^{3}\left(\chi_{-\mathrm{A}}(x) \Delta_{4} \Delta_{\mathrm{A}}^{*} \psi_{+\mathrm{A}}(x)-\psi_{+\mathrm{A}}(x) \Delta_{\mathrm{A}} \Delta_{4}^{*} \chi_{-\mathrm{A}}(x)\right) \\
& \left.-i a \sum_{\mathrm{A}=1}^{3}\left(\chi_{+\mathrm{A}}(x) \Delta_{4} \Delta_{\mathrm{A}}^{*} \psi_{-\mathrm{A}}(x)-\psi_{-\mathrm{A}}(x) \Delta_{\mathrm{A}} \Delta_{4}^{*} \chi_{+\mathrm{A}}(x)\right)\right],
\end{aligned}
$$

with the explicit form of $P_{\mu}^{\prime}$ in appendix C.3 obeying the algebra same as (2.48). The fermion fields were combined as

$$
\Psi^{T}=\left(\psi_{+1}, \cdots, \psi_{+4},-\chi_{+1}, \chi_{+2}, \chi_{+3}, \frac{1}{2} \eta_{+}, \psi_{-1}, \cdots, \psi_{-4},-\chi_{-1}, \chi_{-2}, \chi_{-3}, \frac{1}{2} \eta_{-}\right)
$$

The situation is parallel to the four-dimensional $\mathcal{N}=2$ case. Although the surplus modes still remain in the four-dimensional model even after the modification, they are removed by doing the dimensional reduction with respect to the fourth direction $x_{4}$. As a result, we obtain $\mathcal{N}=8 \mathrm{SYM}$ theory in three dimensions as well as that in two dimensions by reducing further. Thus, we can construct the $\mathcal{N}=8$ lattice models in two and three dimensions, which are free from the surplus modes.

\section{Dimensionally Reduced $\mathcal{N}=8$ Models in Two and Three Dimensions}

\section{$5.13 \mathrm{D} \mathcal{N}=8$ Case}

After the dimensional reduction with respect to $x_{4}$, the slightly modified model in section 4.2 leads a lattice model for three-dimensional $\mathcal{N}=8 \mathrm{SYM}$. The action $S_{3 D \mathcal{N}=8}^{\mathrm{LAT}}+\Delta S^{\prime}$ is expressed as

$$
\begin{aligned}
S_{3 D \mathcal{N}=8}^{\mathrm{LAT}}=Q_{+} Q_{-} \frac{1}{2 g_{0}^{2}} \sum_{x} \operatorname{tr}\left[-i \vec{B}(x) \cdot\left(\overrightarrow{\Phi^{\prime}}(x)+\Delta \overrightarrow{\Phi^{\prime}}(x)\right)\right. \\
\quad-\frac{1}{3} \sum_{\mathrm{A}, \mathrm{B}, \mathrm{C}=1}^{3} \varepsilon_{\mathrm{ABC}} B_{\mathrm{A}}(x)\left[B_{\mathrm{B}}(x), B_{\mathrm{C}}(x)\right] \\
\left.-\sum_{\mu=1}^{4} \psi_{+\mu}(x) \psi_{-\mu}(x)-\vec{\chi}_{+}(x) \cdot \vec{\chi}_{-}(x)-\frac{1}{4} \eta_{+}(x) \eta_{-}(x)\right],
\end{aligned}
$$

where $x$ represents a three-dimensional lattice site $\left(x_{1}, x_{2}, x_{3}\right)$, and $V(x) \equiv U_{4}(x), V(x)^{\dagger} \equiv$ $U_{-4}(x)$ become site variables. $\overrightarrow{\Phi^{\prime}}(x), \Delta \overrightarrow{\Phi^{\prime}}(x)$ are given by the definition (2.44) and (3.3). $\Delta S^{\prime}$ is expressed as (3.2). The supersymmetry transformation (4.2), (4.3), (4.4) is also dimensionally reduced with respect to $x_{4}$. The result changes the form of the $\mu=4$ part of (4.2) alone. It becomes

$$
\begin{aligned}
& Q_{+} V(x)=i \psi_{+4}(x) V(x), \\
& Q_{-} V(x)=i \psi_{-4}(x) V(x), \\
& Q_{+} \psi_{+4}(x)=i \psi_{+4} \psi_{+4}(x)-i\left(\phi(x)-V(x) \phi(x) V(x)^{\dagger}\right), \\
& Q_{-} \psi_{-4}(x)=i \psi_{-4} \psi_{-4}(x)+i\left(\bar{\phi}(x)-V(x) \bar{\phi}(x) V(x)^{\dagger}\right),
\end{aligned}
$$




$$
\begin{aligned}
Q_{-} \psi_{+4}(x)= & \frac{i}{2}\left\{\psi_{+4}(x), \psi_{-4}(x)\right\}-\frac{i}{2}\left(C(x)-V(x) C(x) V(x)^{\dagger}\right)-\tilde{H}_{4}(x), \\
Q_{+} \psi_{-4}(x)= & \frac{i}{2}\left\{\psi_{+4}(x), \psi_{-4}(x)\right\}-\frac{i}{2}\left(C(x)-V(x) C(x) V(x)^{\dagger}\right)+\tilde{H}_{4}(x), \\
Q_{+} \tilde{H}_{4}(x)= & -\frac{1}{2}\left[\psi_{-4}(x), \phi(x)+V(x) \phi(x) V(x)^{\dagger}\right] \\
& +\frac{1}{4}\left[\psi_{+4}(x), C(x)+V(x) C(x) V(x)^{\dagger}\right] \\
& +\frac{i}{2}\left(\eta_{+}(x)-V(x) \eta_{+}(x) V(x)^{\dagger}\right) \\
& +\frac{i}{2}\left[\psi_{+4}(x), \tilde{H}_{4}(x)\right]+\frac{1}{4}\left[\psi_{+4}(x) \psi_{+4}(x), \psi_{-4}(x)\right] \\
Q_{-} \tilde{H}_{4}(x)= & -\frac{1}{2}\left[\psi_{+4}(x), \bar{\phi}(x)+V(x) \bar{\phi}(x) V(x)^{\dagger}\right] \\
& -\frac{1}{4}\left[\psi_{-4}(x), C(x)+V(x) C(x) V(x)^{\dagger}\right] \\
& -\frac{i}{2}\left(\eta_{-}(x)-V(x) \eta_{-}(x) V(x)^{\dagger}\right) \\
& +\frac{i}{2}\left[\psi_{-4}(x), \tilde{H}_{4}(x)\right]-\frac{1}{4}\left[\psi_{-4}(x) \psi_{-4}(x), \psi_{+4}(x)\right] .
\end{aligned}
$$

Repeating the argument similar to the three-dimensional $\mathcal{N}=4$ case, we can justify the weak field expansion (3.9) and show nonexistence of the surplus modes.

Renormalization After the rescaling

$$
\begin{aligned}
& \phi(x) \rightarrow a \phi(x), \quad \bar{\phi}(x) \rightarrow a \bar{\phi}(x), \quad \vec{B}(x) \rightarrow a \vec{B}(x), \quad C(x) \rightarrow a C(x), \\
& \tilde{H}_{\mu}(x) \rightarrow a^{2} \tilde{H}_{\mu}(x), \quad \tilde{H}_{4}(x) \rightarrow a^{2} \tilde{H}_{4}(x), \quad \vec{H}(x) \rightarrow a^{2} \vec{H}(x), \\
& \psi_{ \pm \mu}(x) \rightarrow a^{3 / 2} \psi_{ \pm \mu}(x), \quad \psi_{ \pm 4}(x) \rightarrow a^{3 / 2} \psi_{ \pm 4}(x), \quad \vec{\chi}_{ \pm}(x) \rightarrow a^{3 / 2} \vec{\chi}_{ \pm}(x), \\
& \eta_{ \pm}(x) \rightarrow a^{3 / 2} \eta_{ \pm}(x),
\end{aligned}
$$

the classical action (5.1) reduces to the desired continuum action for $\mathcal{N}=8 \mathrm{SYM}$ in three dimensions in the continuum limit $a \rightarrow 0$ with $g_{3}^{2} \equiv g_{0}^{2} / a$ fixed. Then, $\mathcal{N}=8$ supersymmetry and rotational symmetry are restored. Now, we argue about the renormalization with noting symmetries of the lattice action (5.1):

- lattice translational symmetry

- $\mathrm{SU}(N)$ gauge symmetry

- supersymmetry $Q_{ \pm}$

- $\mathrm{SU}(2)_{R}$ symmetry of the same form as (4.7)

- interchanging symmetry of $Q_{+} \leftrightarrow Q_{-}$with

$$
\begin{aligned}
& \phi(x) \rightarrow-\bar{\phi}(x), \quad \bar{\phi}(x) \rightarrow-\bar{\phi}(x), \quad \eta_{ \pm}(x) \rightarrow \eta_{\mp}(x), \\
& \tilde{H}_{\mu}(x) \rightarrow-\tilde{H}_{\mu}(x), \quad \tilde{H}_{4}(x) \rightarrow-\tilde{H}_{4}(x), \\
& \psi_{ \pm \mu}(x) \rightarrow \psi_{\mp \mu}(x), \quad \psi_{ \pm 4}(x) \rightarrow \psi_{\mp 4}(x), \quad \vec{B}(x) \rightarrow-\vec{B}(x), \quad \vec{\chi}_{ \pm}(x) \rightarrow-\vec{\chi}_{\mp}(x), \\
& \text { others }\left(U_{\mu}(x), V(x), \vec{H}(x), C(x)\right) \text { not changed. }
\end{aligned}
$$


- cyclic permutation $x \equiv\left(x_{1}, x_{2}, x_{3}\right) \rightarrow \tilde{x} \equiv\left(x_{3}, x_{1}, x_{2}\right)$ as

$$
\begin{aligned}
\left(U_{1}(x), U_{2}(x), U_{3}(x), V(x)\right) & \rightarrow\left(U_{2}(\tilde{x}), U_{3}(\tilde{x}), U_{1}(\tilde{x}), V(\tilde{x})\right) \\
\left(\psi_{ \pm 1}(x), \psi_{ \pm 2}(x), \psi_{ \pm 3}(x), \psi_{ \pm 4}(x)\right) & \rightarrow\left(\psi_{ \pm 2}(\tilde{x}), \psi_{ \pm 3}(\tilde{x}), \psi_{ \pm 1}(\tilde{x}), \psi_{ \pm 4}(\tilde{x})\right) \\
\left(\tilde{H}_{1}(x), \tilde{H}_{2}(x), \tilde{H}_{3}(x), \tilde{H}_{4}(x)\right) & \rightarrow\left(\tilde{H}_{2}(\tilde{x}), \tilde{H}_{3}(\tilde{x}), \tilde{H}_{1}(\tilde{x}), \tilde{H}_{4}(\tilde{x})\right) \\
\left(B_{1}(x), B_{2}(x), B_{3}(x)\right) & \rightarrow\left(B_{2}(\tilde{x}), B_{3}(\tilde{x}), B_{1}(\tilde{x})\right) \\
\left(\chi_{ \pm 1}(x), \chi_{ \pm 2}(x), \chi_{ \pm 3}(x)\right) & \rightarrow\left(\chi_{ \pm 2}(\tilde{x}), \chi_{ \pm 3}(\tilde{x}), \chi_{ \pm 1}(\tilde{x})\right) \\
\left(H_{1}(x), H_{2}(x), H_{3}(x)\right) & \rightarrow\left(H_{2}(\tilde{x}), H_{3}(\tilde{x}), H_{1}(\tilde{x})\right) \\
(\phi(x), \bar{\phi}(x), C(x)) & \rightarrow(\phi(\tilde{x}), \bar{\phi}(\tilde{x}), C(\tilde{x})) \\
\eta_{ \pm}(x) & \rightarrow \eta_{ \pm}(\tilde{x}) .
\end{aligned}
$$

Parallel to the argument given in the three-dimensional $\mathcal{N}=4$ case, operators we have to check are those with the dimension $p=1,2,3$ of the types appearing in Table 1 . The effect of $\Delta S^{\prime}$ is irrelevant in the argument of the renormalization. For $p=1,2$, the four operators $\operatorname{tr}\left(4 \phi \bar{\phi}+C^{2}\right), \operatorname{tr} v^{2}, \operatorname{tr}\left(B_{\mathrm{A}} v\right), \operatorname{tr}\left(B_{\mathrm{A}} B_{\mathrm{B}}\right)$ satisfy the requirement from the $\mathrm{SU}(N)$ gauge invariance and the $\mathrm{SU}(2)_{R}$ symmetry, but all of them conflict with the supersymmetry $Q_{ \pm}$.

Next, to search the $p=3$ operators, it is convenient to utilize the result of $Q_{ \pm^{-}}$ cohomology. Under the assignment of the degrees $\left(q_{+}, q_{-}\right)$to each variable:

$$
\begin{array}{ccc}
U_{\mu}:(0,0) & \vec{B}:(1,1) & \phi:(2,0) \\
\psi_{+\mu}:(1,0) & \vec{\chi}_{+}:(2,1) & \bar{\phi}:(0,2) \\
\psi_{-\mu}:(0,1) & \vec{\chi}_{-}:(1,2) & C:(1,1) \\
\tilde{H}_{\mu}:(1,1) & \vec{H}:(2,2) & \eta_{+}:(2,1) \\
& & \eta_{-}:(1,2)
\end{array}
$$

(here, $\mu=1, \cdots, 4$ ), the result is summarized as follows:

Any gauge invariant and $S U(2)_{R}$ invariant operator $\alpha$ annihilated by $Q_{ \pm}$can be expressed as

$$
\begin{aligned}
\alpha & =\alpha_{0}+Q_{+} \beta_{-}+Q_{+} Q_{-\gamma} \\
& =\alpha_{0}+Q_{-} \beta_{+}+Q_{+} Q_{-} \gamma
\end{aligned}
$$

$\alpha_{0}$ is proportional to the identity, and $\beta_{ \pm}, \gamma$ are gauge invariant. $\beta_{+}\left(\beta_{-}\right)$is a $Q_{+^{-}}\left(Q_{-_{-}}\right)$ closed operator of the degrees $(1,0)((0,1))$, and has nontrivial cohomology with respect to $Q_{+}\left(Q_{-}\right)$. (For a proof of this statement, see appendix C in ref. [1].) Since the mass dimension of $\beta_{ \pm}$is $5 / 2$, gauge invariant candidates for $\beta_{-}$are $\operatorname{tr}\left(v \psi_{-\mu}\right)$ alone. However, they are not $Q_{-}$invariant. Hence, there is no operator of the type $Q_{+} \beta_{-}$. Similar holds for $Q_{-} \beta_{+}$from the $Q_{+} \leftrightarrow Q_{-}$symmetry. $\gamma$ has the dimension two with the $\mathrm{U}(1)_{R}$ neutral, and it must be odd under $Q_{+} \leftrightarrow Q_{-}$from the symmetry requirement (5.4). It leads the six candidates $\operatorname{tr}\left(B_{\mathrm{A}} v\right), \operatorname{tr}\left(B_{\mathrm{A}} C\right)(\mathrm{A}=1,2,3)$ for $\gamma$. Among them, the two combinations $\operatorname{tr}\left[\left(B_{1}+B_{2}+B_{3}\right) v\right], \operatorname{tr}\left[\left(B_{1}+B_{2}+B_{3}\right) C\right]$ survive by imposing the requirement (5.5). Furthermore, the $\mathrm{SU}(2)_{R}$ invariance of $Q_{+} Q_{-} \gamma$ singles out the former. Thus, we can 
conclude that the $p=3$ operator

$$
\begin{aligned}
Q_{+} Q_{-} \operatorname{tr}\left[\left(B_{1}+B_{2}+B_{3}\right) v\right]= & \sum_{\mathrm{A}=1}^{3} \operatorname{tr}\left[\left(\frac{1}{2}\left[C, B_{\mathrm{A}}\right]+H_{\mathrm{A}}+\sum_{\mathrm{B}, \mathrm{C}=1}^{3} \frac{1}{2} \varepsilon_{\mathrm{ABC}}\left[B_{\mathrm{B}}, B_{\mathrm{C}}\right]\right) v\right. \\
& \left.+B_{\mathrm{A}}\left(-\frac{1}{2}[v, C]+\tilde{H}_{4}\right)+\chi_{+\mathrm{A}} \psi_{-4}-\chi_{-\mathrm{A}} \psi_{+4}\right],
\end{aligned}
$$

which is the only one consistent to the symmetries possessed by the lattice action, is possibly generated as the radiative corrections. In order to reach the desired continuum theory with the full $\mathcal{N}=8$ supersymmetry restored, it is necessary to add a counter term corresponding the above operator to cancel it with the radiative corrections by fine-tuning.

\section{$5.22 \mathrm{D} \mathcal{N}=8$ Case}

A lattice theory for $\mathcal{N}=8 \mathrm{SYM}$ in two dimensions is obtained by the further dimensional reduction from the three-dimensional $\mathcal{N}=8$ model (5.1) with respect to $x_{3}$. The action takes the same form as $(5.1)+\Delta S^{\prime}$, but now $x$ stands for a two-dimensional lattice site $\left(x_{1}, x_{2}\right)$. Also, the variables $W(x) \equiv U_{3}(x), W(x)^{\dagger}=U_{-3}(x)$ become sitting at sites. $\overrightarrow{\Phi^{\prime}}$, $\Delta \overrightarrow{\Phi^{\prime}}$ are defined by $(2.44)$ and (3.23).

When considering fluctuations around the minimum of the action, the weak field expansion of the form (3.25) is justified, and no surplus mode appears. The naive continuum limit $a \rightarrow 0$ with $g_{2}^{2} \equiv g_{0}^{2} / a^{2}$ fixed leads the desired continuum action after a suitable rescaling of lattice fields corresponding to the order (4.6). Because any operators of the types $\varphi$, $\varphi \varphi$ with $p=1,2$ in Table 1 do not satisfy the requirement from the $Q_{ \pm}$supersymmetry, the loop corrections are not allowed to generate any relevant or marginal operators except the identity. It means restoration of the full supersymmetry and the rotational invariance in the continuum limit at the quantum level without fine-tuning.

\section{Summary and Discussions}

In this paper, first we have discussed on naive lattice models for $\mathcal{N}=2,4 \mathrm{SYM}$ theories in four-dimensions constructed based on the 'TFT, BTFT formulations' along the same line to the papers $[1,2]$. A problem of the degenerate vacua encountered in the paper [1] is resolved by extending some fields and soaking up would-be zero-modes in the continuum limit. We see that there appear surplus zero-modes carrying nonzero momenta in the kinetic terms for both of bosons and fermions in addition to the ordinary zero-momentum mode. The appearence of the surplus modes seems to be related to the fact that our construction of a lattice counter part of the quantity $\int F \wedge F$ is not exactly topological on the lattice.

By dimensional reduction of a slightly modified version of the four-dimensional models, we have constructed two- and three-dimensional models with $\mathcal{N}=4,8$ supersymmetry, which are completely free from the surplus modes. These models flow into the desired continuum theories with three and one parameters fine-tuned for three-dimensional $\mathcal{N}=$ 4, 8 cases, while no fine-tuning is required in the two-dimensional cases. This is a main result of this paper. 
After building the models, it is natural to consider actual numerical computation by utilizing the lattice actions presented here. A first step toward the end will be to examine the positivity of the fermion determinant similarly as in refs. [15] for the case of lattice models based on the idea of the deconstruction [9]. Also, for the numerical study, it would be attractive to apply the method of density matrix renormalization group [16] to our lattice models via the Hamiltonian formulation.

As for four-dimensional $\mathcal{N}=4$ theory, it is believed that it has no anomaly due to the ultra-violet finiteness [17] differently from the $\mathcal{N}=2$ case. Suppose that we may not consider about the problem of the surplus modes. Then, since the supersymmetry $Q_{ \pm}$of the $\mathcal{N}=4$ lattice model enhances to the full $\mathcal{N}=4$ supersymmetry in the naive continuum limit, only the lattice artifact of $O(a)$ breaks the full 16 supercharges down to the two $Q_{ \pm}$ and violates the ultra-violet finiteness. It induces loop divergence as $a \rightarrow 0$. Thus, the radiative corrections seem to have the structure something like

$$
\text { (lattice artifact of } O(a)) \times(\text { loop divergence as } a \rightarrow 0) \text {, }
$$

which are somewhat subtle to say whether they survive in the continuum limit or not. It would be interesting to do an explicit perturbative analysis for the four-dimensional $\mathcal{N}=4$ lattice model, and to see whether the radiative corrections induce relevant or marginal operators to the action.

$\mathcal{N}=8$ SYM theories in two and three dimensions have been proposed as matrix string models which serve nonperturbative definitions of superstring theory [18]. It would be meaningful to do nonperturbative investigation of the corresponding lattice models presented here from the viewpoint of string theory as well as the field-theoretical interest.

\section{Acknowledgments}

The author would like to thank S. Catterall, K. Fujikawa, J. Giedt, M. Kato, I. Kanamori, N. Kawamoto, Y. Kikukawa, K. Nagata, M. Sakamoto, H. So, T. Sugihara and T. Takimi for variable discussions and useful conversations.

\section{A. On Solutions of Eq. (2.10)}

In this appendix, we show that solutions of the equation for $U, V \in \mathrm{SU}(N)$

$$
e^{-i \varphi}(U+V)+e^{i \varphi}\left(U^{\dagger}+V^{\dagger}\right)=\left(\frac{1}{N} \operatorname{tr}\left[e^{-i \varphi}(U+V)+e^{i \varphi}\left(U^{\dagger}+V^{\dagger}\right)\right]\right) \mathbf{1}_{N}
$$

are not unique and distribute continuously from $U=V=1$. It leads that the solutions of (2.10) also distribute continuously from $U_{\mu \nu}(x)=1$.

Diagonalizing $U, V$ as

$$
U=\Omega_{U}\left(\begin{array}{ccc}
e^{i u_{1}} & & \\
& \ddots & \\
& & e^{i u_{N}}
\end{array}\right) \Omega_{U}^{\dagger}, \quad V=\Omega_{V}\left(\begin{array}{ccc}
e^{i v_{1}} & & \\
& \ddots & \\
& & e^{i v_{N}}
\end{array}\right) \Omega_{V}^{\dagger}
$$


with $\Omega_{U}, \Omega_{V} \in \mathrm{SU}(N)$, eq. (A.1) can be expressed in the form

$$
\left(\begin{array}{ccc}
\cos \left(u_{1}-\varphi\right) & & \\
& \ddots & \\
& & \cos \left(u_{N}-\varphi\right)
\end{array}\right)+\Omega\left(\begin{array}{ccc}
\cos \left(v_{1}-\varphi\right) & \\
& \ddots & \\
& & \cos \left(v_{N}-\varphi\right)
\end{array}\right) \Omega^{\dagger}=c \mathbf{1}_{N}
$$

with $c$ some real constant. Since $\Omega \equiv \Omega_{U}^{\dagger} \Omega_{V}$ is adjoint action transforming from a diagonal matrix to a diagonal matrix, it must belong to the Weyl group of $\mathrm{SU}(N)$, i.e. $\Omega$ leads even permutations of diagonal entries. Thus eq. (A.3) is equivalent to the following set of equations:

$$
\left\{\begin{array}{cr}
\cos \left(u_{1}-\varphi\right)+\cos \left(v_{i(1)}-\varphi\right)= & c \\
\vdots & \vdots \\
\cos \left(u_{N}-\varphi\right)+\cos \left(v_{i(N)}-\varphi\right)= & c
\end{array}\right.
$$

with an even permutation: $(1, \cdots, N) \rightarrow(i(1), \cdots, i(N))$.

Let us take a parametrization

$$
u_{1}+\cdots+u_{N}=0, \quad v_{1}+\cdots+v_{N}=0
$$

Because the permutation is just renaming the variables, we may consider the case $i(1)=$ $1, \cdots, i(N)=N$ without loss of generality. Then the equation can be written as

$$
\begin{array}{cc}
\cos \left(u_{1}-\varphi\right)+\cos \left(v_{1}-\varphi\right) & =\cos \left(u_{2}-\varphi\right)+\cos \left(v_{2}-\varphi\right), \\
\cos \left(u_{1}-\varphi\right)+\cos \left(v_{1}-\varphi\right) & =\cos \left(u_{3}-\varphi\right)+\cos \left(v_{3}-\varphi\right), \\
\vdots & \vdots \\
\cos \left(u_{1}-\varphi\right)+\cos \left(v_{1}-\varphi\right)=\cos \left(u_{N}-\varphi\right)+\cos \left(v_{N}-\varphi\right) .
\end{array}
$$

Let us consider the case $u_{1}=\cdots=u_{N-1} \equiv u, v_{1}=\cdots=v_{N-1} \equiv v$. From (A.5), $u_{N}=-(N-1) u, v_{N}=-(N-1) v$. Among (A.6), the equation

$$
f(u)=-f(v)
$$

with

$$
f(x) \equiv \cos (x-\varphi)-\cos ((N-1) x+\varphi)
$$

only remains nontrivial. When $|x|$ is small, $f(x)$ is monotonically increasing $\left(f^{\prime}(x)>0\right)$ for

$$
(N-1)|x|<\varphi<\pi-(N-1)|x| .
$$

Thus, there exist solutions of (A.7) continuously connecting from the trivial one $u=v=0$ for $\varphi$ satisfying (A.9) with $|x|=\max (|u|,|v|)$.

Suppose that we consider the case $U=U_{14}, V=U_{23}$ and $U_{1}=U_{1}\left(x_{1}, x_{4}\right)$ ( $U_{1}$ depends only on $x_{1}$ and $\left.x_{4}\right), U_{2}=U_{2}\left(x_{2}, x_{3}\right)$ ( $U_{2}$ depends only on $x_{2}$ and $\left.x_{3}\right), U_{3}=U_{4}=1$, for example. Then, $U_{24}(x)=U_{34}(x)=U_{12}(x)=U_{31}(x)=1$, and the equations

$$
\Phi_{2}(x)+\Delta \Phi_{2}(x) \propto \mathbf{1}_{N}, \quad \Phi_{3}(x)+\Delta \Phi_{3}(x) \propto \mathbf{1}_{N}
$$

are trivially satisfied. The remaining $\Phi_{1}(x)+\Delta \Phi_{1}(x) \propto \mathbf{1}_{N}$ has solutions continuously connecting from $U_{14}(x)=U_{23}(x)=1$. 


\section{B. Resolution of Vacuum Degeneracy}

Here we show that the minimum

$$
\vec{\Phi}(x)+\Delta \vec{\Phi}(x)=0
$$

of the gauge field action is uniquely given by the configuration $U_{\mu \nu}(x)=1$ when the parameter $r=\cot \varphi$ ranging as

$$
0<\varphi \leq \frac{\pi}{2 N}
$$

To do that, it is sufficient to see that the equation for $U, V \in \mathrm{SU}(N)$ :

$$
e^{-i \varphi}(-2+U+V)+e^{i \varphi}\left(-2+U^{\dagger}+V^{\dagger}\right)=0
$$

has the unique solution $U=V=1$ when $\varphi$ satisfies (B.2).

Diagonalizing $U, V$ as

$$
U=\Omega_{U}\left(\begin{array}{ccc}
e^{i u_{1}} & & \\
& \ddots & \\
& & e^{i u_{N}}
\end{array}\right) \Omega_{U}^{\dagger}, \quad V=\Omega_{V}\left(\begin{array}{ccc}
e^{i v_{1}} & & \\
& \ddots & \\
& & e^{i v_{N}}
\end{array}\right) \Omega_{V}^{\dagger}
$$

with $\Omega_{U}, \Omega_{V} \in \mathrm{SU}(N)$, eq. (B.3) can be expressed in the form

$$
\Omega\left(\begin{array}{ccc}
\cos \left(v_{1}-\varphi\right) & & \\
& \ddots & \\
& & \cos \left(v_{N}-\varphi\right)
\end{array}\right) \Omega^{\dagger}=2 \cos \varphi-\left(\begin{array}{ccc}
\cos \left(u_{1}-\varphi\right) & & \\
& \ddots & \\
& & \cos \left(u_{N}-\varphi\right)
\end{array}\right)
$$

Since $\Omega \equiv \Omega_{U}^{\dagger} \Omega_{V}$ is adjoint action transforming from a diagonal matrix to a diagonal matrix, it must belong to the Weyl group of $\mathrm{SU}(N)$, i.e. $\Omega$ leads even permutations of diagonal entries. Thus eq. (B.5) is equivalent to the following set of equations:

$$
\left\{\begin{array}{cc}
\cos \left(v_{i(1)}-\varphi\right) & 2 \cos \varphi-\cos \left(u_{1}-\varphi\right) \\
\vdots & \vdots \\
\cos \left(v_{i(N)}-\varphi\right) & =2 \cos \varphi-\cos \left(u_{N}-\varphi\right)
\end{array}\right.
$$

with an even permutation: $(1, \cdots, N) \rightarrow(i(1), \cdots, i(N))$.

Let us consider the equations for an arbitrarily fixed permutation $i$. We may take the following parametrizations:

$$
\begin{aligned}
& -\pi<u_{1}, \cdots, u_{N-1} \leq \pi, \quad u_{N}=-u_{1}-\cdots-u_{N-1} \\
& -\pi<v_{i(1)}, \cdots, v_{i(N-1)} \leq \pi, \quad v_{i(N)}=-v_{i(1)}-\cdots-v_{i(N-1)} .
\end{aligned}
$$

Eq. (B.6) can be written as

$$
\cos \left(\frac{u_{k}+v_{i(k)}}{2}-\varphi\right) \cos \left(\frac{u_{k}-v_{i(k)}}{2}\right)=\cos \varphi \quad(k=1, \cdots, N) .
$$


In eqs. (B.8), $\left|\cos \left(\frac{u_{k}-v_{i(k)}}{2}\right)\right| \leq 1$ requires

$$
\left|\cos \left(\frac{u_{k}+v_{i(k)}}{2}-\varphi\right)\right| \geq \cos \varphi
$$

which means

$$
\pi n \leq \frac{u_{k}+v_{i(k)}}{2} \leq 2 \varphi+\pi n \quad(n=0, \pm 1, \cdots) .
$$

The case $k=1, \cdots, N-1$ in eq. (B.10) First consider the case $k=1, \cdots, N-1$. From the parametrization (B.7), the relation (B.10) is realized by the following three cases:

$$
\begin{aligned}
& 0 \leq \frac{u_{k}+v_{i(k)}}{2} \leq 2 \varphi, \\
& \frac{u_{k}+v_{i(k)}}{2}=\pi, \\
& -\pi<\frac{u_{k}+v_{i(k)}}{2} \leq 2 \varphi-\pi .
\end{aligned}
$$

The second possibility (B.12) means $u_{k}=v_{i(k)}=\pi$, but it is not consistent to (B.8). Also, the third (B.13) leads $\cos \left(\frac{u_{k}+v_{i(k)}}{2}\right)<0$, which requires

$$
\cos \left(\frac{u_{k}-v_{i(k)}}{2}\right)<0
$$

from the consistency with eq. (B.8). Then, we obtain

$$
\frac{\pi}{2}<\frac{u_{k}-v_{i(k)}}{2}<\pi \quad \text { or } \quad-\pi<\frac{u_{k}-v_{i(k)}}{2}<-\frac{\pi}{2}
$$

from (B.14). However, it is easy to see that (B.13) and (B.15) are not compatible with each other. Thus, only the possibility (B.11) remains.

The case $k=N$ in eq. (B.10) Next, we see the case $k=N$, which reads

$$
-2 \varphi+\pi n \leq \frac{1}{2}\left(u_{1}+\cdots+u_{N-1}+v_{i(1)}+\cdots+v_{i(N-1)}\right) \leq \pi n
$$

with $n=0, \pm 1, \cdots$. On the other hand, summing (B.11) over $k=1, \cdots, N-1$ gives

$$
0 \leq \frac{1}{2}\left(u_{1}+\cdots+u_{N-1}+v_{i(1)}+\cdots+v_{i(N-1)}\right) \leq 2(N-1) \varphi .
$$

From these two equations, we obtain

$$
\frac{1}{2}\left(u_{1}+\cdots+u_{N-1}+v_{i(1)}+\cdots+v_{i(N-1)}\right)= \begin{cases}0, \pi \frac{N-1}{N} & \text { for } \varphi=\frac{\pi}{2 N} . \\ 0 & \text { for } 0<\varphi<\frac{\pi}{2 N} .\end{cases}
$$

In the case $\frac{1}{2}\left(u_{1}+\cdots+u_{N-1}+v_{i(1)}+\cdots+v_{i(N-1)}\right)=\pi \frac{N-1}{N}$ for $\varphi=\frac{\pi}{2 N}$, owing to (B.11) we have

$$
u_{1}+v_{i(1)}=\cdots=u_{N-1}+v_{i(N-1)}=\frac{2 \pi}{N} .
$$


(B.8) becomes $\cos \left(\frac{u_{k}-v_{i(k)}}{2}\right)=1$ for $k=1, \cdots, N-1$ leading

$$
u_{k}=v_{i(k)} \quad \text { for } k=1, \cdots, N-1 .
$$

Thus, we get

$$
u_{1}=\cdots=u_{N-1}=v_{i(1)}=\cdots=v_{i(N-1)}=\frac{\pi}{N},
$$

however which is not consistent to the $k=N$ part of the eq. (B.8):

$$
\cos \left[\frac{1}{2}\left(u_{1}+\cdots+u_{N-1}+v_{i(1)}+\cdots+v_{i(N-1)}\right)+\frac{\pi}{2 N}\right]=\cos \frac{\pi}{2 N} .
$$

For the remaining case $\frac{1}{2}\left(u_{1}+\cdots+u_{N-1}+v_{i(1)}+\cdots+v_{i(N-1)}\right)=0$, we have

$$
u_{1}+v_{i(1)}=\cdots=u_{N-1}+v_{i(N-1)}=0
$$

from (B.11), and then (B.8) becomes $\cos \left(\frac{u_{k}-v_{i(k)}}{2}\right)=1$ for $k=1, \cdots, N-1$ meaning

$$
u_{k}=v_{i(k)} \quad \text { for } k=1, \cdots, N-1 .
$$

Eqs. (B.23) and (B.24) lead

$$
u_{1}=\cdots=u_{N-1}=v_{i(1)}=\cdots=v_{i(N-1)}=0
$$

to give $u_{N}=v_{i(N)}=0$. It is compatible to the possibility (B.11).

Therefore, the analysis shows that eqs. (B.8) allow the solution $u_{1}=\cdots=u_{N}=v_{1}=$ $\cdots=v_{N}=0$ only. We conclude that the equation (B.3) has the unique solution $U=V=1$ with choice of $r=\cot \varphi$ in the range $0<\varphi \leq \frac{\pi}{2 N}$.

\section{Explicit Form of the matrices $\gamma_{\mu}, P_{\mu}, P_{\mu}^{\prime}$}

We present the explicit form of the matrices $\gamma_{\mu}$ and $P_{\mu}$ appearing in the naive lattice models of four-dimensional $\mathcal{N}=2,4$ theories as well as $P_{\mu}^{\prime}$ in their slightly modified versions. In what follows, $\sigma_{1}, \sigma_{2}$ and $\sigma_{3}$ represent the Pauli matrices.

\section{C.1 Naive Model for four-dimensional $\mathcal{N}=2$ Theory}

First, in the naive model for $\mathcal{N}=2, \gamma_{\mu}$ is written as the form

$$
\gamma_{\mu}=-i\left(\begin{array}{cc}
0 & \xi_{\mu} \\
\xi_{\mu}^{T} & 0
\end{array}\right)
$$

where

$$
\xi_{1}=\left(\begin{array}{c}
\sigma_{1} \\
-i \sigma_{2}
\end{array}\right), \quad \xi_{2}=\left(\begin{array}{c}
{ }^{-} \sigma_{3} \\
\mathbf{1}_{2}
\end{array}\right), \quad \xi_{3}=\left(\begin{array}{cc}
i \sigma_{2} & \\
& \sigma_{1}
\end{array}\right), \quad \xi_{4}=\left(\begin{array}{cc}
-\mathbf{1}_{2} & \\
& -\sigma_{3}
\end{array}\right) .
$$

Also, $P_{\mu}$ are written as

$$
P_{\mu}=\left(\begin{array}{cc}
0 & \nu_{\mu} \\
\nu_{\mu} & 0
\end{array}\right)=\sigma_{1} \otimes \nu_{\mu} \quad \text { for } \mu=1, \cdots, 3, \quad P_{4}=\sigma_{2} \otimes \mathbf{1}_{4}
$$

with

$$
\nu_{1}=\left(\begin{array}{cc}
0 & \sigma_{2} \\
\sigma_{2} & 0
\end{array}\right), \quad \nu_{2}=\left(\begin{array}{cc}
0 & -i \mathbf{1}_{2} \\
i \mathbf{1}_{2} & 0
\end{array}\right), \quad \nu_{3}=\left(\begin{array}{cc}
-\sigma_{2} & 0 \\
0 & \sigma_{2}
\end{array}\right)
$$




\section{C.2 Slightly Modified Model for four-dimensional $\mathcal{N}=2$ Theory}

In the slightly modified model for four-dimensional $\mathcal{N}=2$ theory, the gamma matrices are same as (C.1), (C.2) in the naive model, while $P_{\mu}^{\prime}$ are of the form

$$
P_{\mu}^{\prime}=-i\left(\begin{array}{cc}
0 & \nu_{\mu}^{\prime} \\
-\nu_{\mu}^{\prime T} & 0
\end{array}\right)
$$

with

$$
\nu_{1}^{\prime}=\left(\begin{array}{cc}
0 & i \sigma_{2} \\
\sigma_{1} & 0
\end{array}\right), \quad \nu_{2}^{\prime}=\left(\begin{array}{cc}
0 & \mathbf{1}_{2} \\
-\sigma_{3} & 0
\end{array}\right), \quad \nu_{3}^{\prime}=\left(\begin{array}{cc}
-i \sigma_{2} & 0 \\
0 & \sigma_{1}
\end{array}\right), \quad \nu_{4}^{\prime}=\mathbf{1}_{4} .
$$

C.3 Naive Model for four-dimensional $\mathcal{N}=4$ Theory

$\gamma_{\mu}$ appearing in the naive model for $\mathcal{N}=4$ take the form of (C.1) with

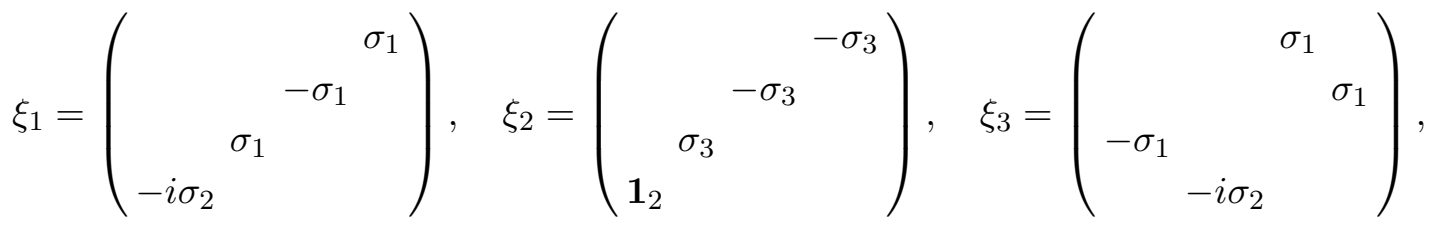

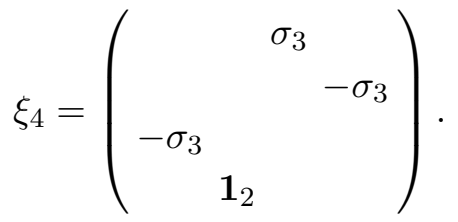

$$
P_{\mu}=-i\left(\begin{array}{cc}
0 & \omega_{\mu} \\
-\omega_{\mu}^{T} & 0
\end{array}\right)
$$

where

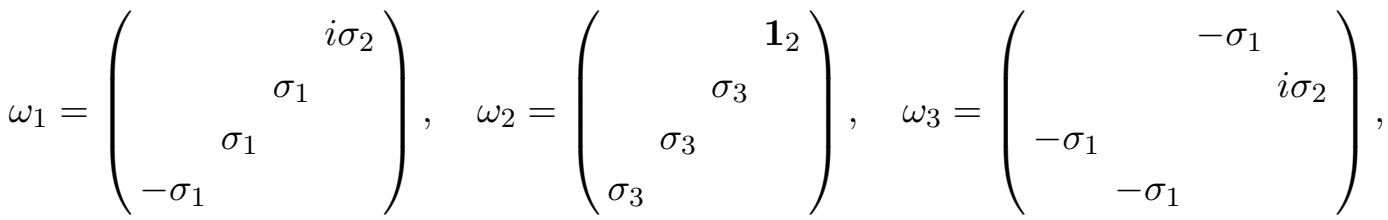

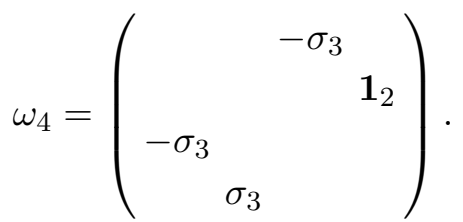

\section{C.4 Slightly Modified Model for four-dimensional $\mathcal{N}=4$ Theory}

For the slightly modified model in the case of $\mathcal{N}=4$ theory, $\gamma_{\mu}$ are given by (C.1), (C.7), and $P_{\mu}^{\prime}$ are written as

$$
P_{\mu}^{\prime}=-i\left(\begin{array}{cc}
0 & \omega_{\mu}^{\prime} \\
-\omega_{\mu}^{\prime T} & 0
\end{array}\right)
$$


where

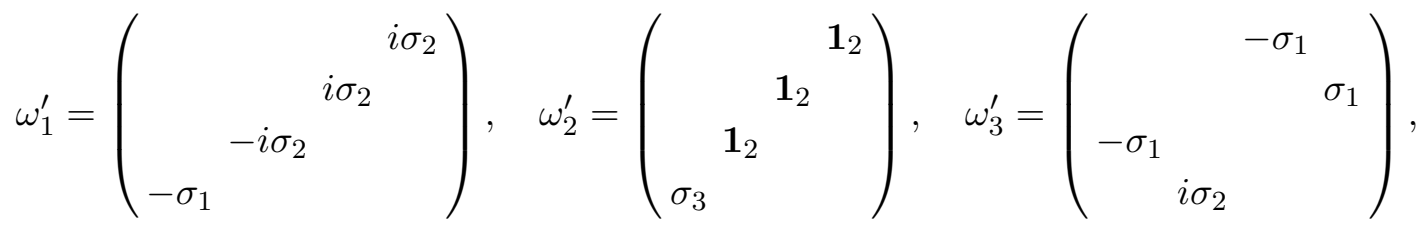

$$
\begin{aligned}
& \omega_{4}^{\prime}=\left(\begin{array}{ccc} 
& & -\sigma_{3} \\
& & \mathbf{1}_{2} \\
-\sigma_{3} & & \\
\sigma_{3} &
\end{array}\right) \text {. }
\end{aligned}
$$

\section{References}

[1] F. Sugino, A lattice formulation of super Yang-Mills theories with exact supersymmetry, JHEP 0401 (2004) 015, [hep-lat/0311021].

[2] F. Sugino, Super Yang-Mills theories on the two-dimensional lattice with exact supersymmetry, JHEP 0403 (2004) 067, [hep-lat/0401017].

[3] E. Witten, Topological Quantum Field Theory, Commun. Math. Phys. 117 (1988) 353; Introduction To Cohomological Field Theories, Int. J. Mod. Phys. A 6 (1991) 2775.

[4] C. Vafa and E. Witten, A Strong coupling test of S duality, Nucl. Phys. B 431 (1994) 3, [hep-th/9408074].

R. Dijkgraaf and G. W. Moore, Balanced topological field theories, Commun. Math. Phys. 185 (1997) 411, [hep-th/9608169].

M. Blau and G. Thompson, Aspects of $N(T) \geq 2$ topological gauge theories and D-branes, Nucl. Phys. B 492 (1997) 545, [hep-th/9612143].

J. M. F. Labastida and C. Lozano, Mathai-Quillen formulation of twisted $N=4$ supersymmetric gauge theories in four dimensions, Nucl. Phys. B 502 (1997) 741, [hep-th/9702106].

[5] S. Catterall, Lattice supersymmetry and topological field theory, JHEP 0305 (2003) 038, [hep-lat/0301028].

S. Catterall and S. Ghadab, Lattice sigma models with exact supersymmetry, JHEP 0405 (2004) 044, [hep-lat/0311042].

A. D'Adda, I. Kanamori, N. Kawamoto and K. Nagata, Twisted superspace on a lattice, [hep-lat/0406029].

J. Giedt and E. Poppitz, Lattice supersymmetry, superfields and renormalization, JHEP 0409 (2004) 029, [hep-th/0407135].

[6] S. Catterall and S. Karamov, Exact lattice supersymmetry: the two-dimensional $N=2$

Wess-Zumino model, Phys. Rev. D 65 (2002) 094501, [hep-lat/0108024]; A lattice study of the two-dimensional Wess Zumino model, Phys. Rev. D 68 (2003) 014503,

[hep-lat/0305002].

Y. Kikukawa and Y. Nakayama, Nicolai mapping vs. exact chiral symmetry on the lattice, Phys. Rev. D 66 (2002) 094508, [hep-lat/0207013].

K. Fujikawa, $N=2$ Wess-Zumino model on the $d=2$ Euclidean lattice, Phys. Rev. D 66 (2002) 074510, [hep-lat/0208015]. 
M. Bonini and A. Feo, Wess-Zumino model with exact supersymmetry on the lattice, JHEP 0409 (2004) 011, [hep-lat/0402034].

A. Kirchberg, J. D. Lange and A. Wipf, From the Dirac operator to Wess-Zumino models on spatial lattices, [hep-th/0407207].

[7] K. Itoh, M. Kato, H. Sawanaka, H. So and N. Ukita, Novel approach to super Yang-Mills theory on lattice: Exact fermionic symmetry and 'Ichimatsu' pattern, JHEP 0302 (2003) 033, [hep-lat/0210049].

M. Harada and S. Pinsky, $N=(1,1)$ super Yang-Mills on a $(2+1)$ dimensional transverse lattice with one exact supersymmetry, Phys. Lett. B 567 (2003) 277, [hep-lat/0303027]; A solution to the fermion doubling problem for supersymmetric theories on the transverse lattice, [hep-lat/0408026].

[8] D. B. Kaplan, E. Katz and M. Ünsal, Supersymmetry on a spatial lattice, JHEP 0305 (2003) 037, [hep-lat/0206019].

[9] A. G. Cohen, D. B. Kaplan, E. Katz and M. Ünsal, Supersymmetry on a Euclidean spacetime lattice. I: A target theory with four supercharges, JHEP 0308 (2003) 024, [hep-lat/0302017]; Supersymmetry on a Euclidean spacetime lattice. II: Target theories with eight supercharges, JHEP 0312 (2003) 031, [hep-lat/0307012].

[10] J. Nishimura, S. J. Rey and F. Sugino, Supersymetry on the noncommutative lattice, JHEP 0302 (2003) 032, [hep-lat/0301025].

J. Giedt, E. Poppitz and M. Rozali, Deconstruction, lattice supersymmetry, anomalies and branes, JHEP 0303 (2003) 035, [hep-th/0301048].

M. Ünsal, Regularization of non-commutative SYM by orbifolds with discrete torsion and SL (2,Z) duality, [hep-th/0409106].

[11] I. Montvay, Supersymmetric Yang-Mills theory on the lattice, Int. J. Mod. Phys. A 17 (2002) 2377, [hep-lat/0112007].

A. Feo, Predictions and recent results in SUSY on the lattice, [hep-lat/0410012].

[12] S. Elitzur, E. Rabinovici and A. Schwimmer, Supersymmetric Models On The Lattice, Phys. Lett. B 119 (1982) 165.

[13] M. Lüscher, Topology and the axial anomaly in abelian lattice gauge theories, Nucl. Phys. B 538 (1999) 515, [hep-lat/9808021].

T. Fujiwara, H. Suzuki and K. Wu, Axial anomaly in lattice Abelian gauge theory in arbitrary dimensions, Phys. Lett. B 463 (1999) 63, [hep-lat/9906016]; Non-commutative differential calculus and the axial anomaly in Abelian lattice gauge theories, Nucl. Phys. B 569 (2000) 643, [hep-lat/9906015].

[14] N. Seiberg and E. Witten, Electric - magnetic duality, monopole condensation, and confinement in N=2 supersymmetric Yang-Mills theory, Nucl. Phys. B 426 (1994) 19 [Erratum-ibid. B 430 (1994) 485], [hep-th/9407087].

[15] J. Giedt, Non-positive fermion determinants in lattice supersymmetry, Nucl. Phys. B 668 (2003) 138, [hep-lat/0304006]; The fermion determinant in (4,4) 2d lattice super-Yang-Mills, Nucl. Phys. B 674 (2003) 259, [hep-lat/0307024]; Deconstruction, 2d lattice Yang-Mills, and the dynamical lattice spacing, [hep-lat/0312020].

[16] T. Sugihara, Density matrix renormalization group in a two-dimensional $\lambda \phi^{4}$ Hamiltonian lattice model, JHEP 0405 (2004) 007, [hep-lat/0403008]. 
[17] S. Mandelstam, Light Cone Superspace And The Ultraviolet Finiteness Of The N=4 Model, Nucl. Phys. B 213 (1983) 149.

[18] R. Dijkgraaf, E. Verlinde and H. Verlinde, Matrix string theory, Nucl. Phys. B 500 (1997) 43, [hep-th/9703030].

T. Banks and N. Seiberg, Strings from matrices, Nucl. Phys. B 497 (1997) 41, [hep-th/9702187]. 